The Free Internet Journal

for Organic Chemistry
Paper

Arkivoc 2017, part v, 314-326

Organic Chemistry

\title{
Synthesis of substituted imid(thi)azolidines by [3+2] cycloaddition of aziridines with nitriles(isothiocyanates) via visible light photocatalysis
}

\begin{abstract}
Qianwen Ye, ${ }^{\mathrm{a}}$ Xiaoliang Xu, ${ }^{* a}$ Dongping Cheng, ${ }^{\text {b }}$ Baochuan Guan, ${ }^{\mathrm{a}}$ Hongfeng $\mathrm{Ye}^{\mathrm{a}}{ }^{\mathrm{a}}$ and Xiaonian Li*a
${ }^{a}$ College of Chemical Engineering, Zhejiang University of Technology, Hangzhou 310014, P. R. China

${ }^{b}$ College of Pharmaceutical Science, Zhejiang University of Technology, Hangzhou 310014, P. R. China

Email:xuxiaoliang@zjut.edu.cn, chengdp@zjut.edu.cn, xnli@zjut.edu.cn
\end{abstract}

Received 07-02-2017

Accepted 10-22-2017

Published on line 11-19-2017

\section{Abstract}

The [3+2] cycloaddition reaction of aziridines with nitriles (isothiocyanates) has been developed using visible light photocatalysis. Two types of five-membered heterocyclic compounds, imidazolidines and thiazolidines, were synthesized in mild conditions. An oxidative quenching cycle mechanism is probably involved.

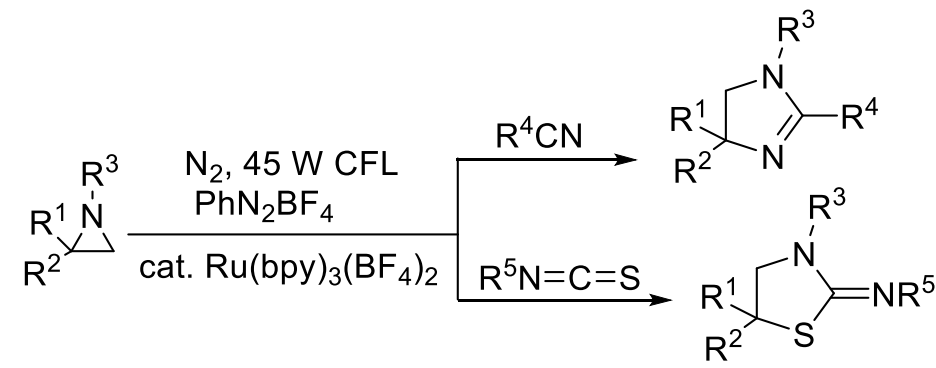

Keywords: Aziridines, nitriles, isothiocyanates, [3+2] cycloaddition, photocatalysis 


\section{Introduction}

Photoredox catalysis by means of visible light is considered an ideal route for green, economic and abundant chemical synthesis. Different from many conventional reagents, visible light can be used as a type of renewable source. ${ }^{1-5}$ Since it was proposed by Ciamician in 1912, this concept has been almost silent for half a century or more. ${ }^{6}$ It was not until 2008 that MacMillan firstly reported $\alpha$-alkylation of aldehydes and ketones by photocatalysis, which attracted considerable attention from chemists. ${ }^{7}$ Subsequently, many research groups have made extensive and outstanding contributions in this field ${ }^{1}$ and photocatalysis has evolved into an important means in organic synthesis.

Imidazolines and thiazolines are important heterocyclic systems which have a wide range of applications in bio-activities ${ }^{8-9}$ and pharmaceuticals. ${ }^{10-11}$ Some derivatives are used for the synthesis of anti-inflammatory, antioxidant, anti-nociceptive and anticarcinogenic drugs. Additionally, imidazolines have been used as metal complex ligands ${ }^{12}$ and auxiliary groups ${ }^{13}$ in asymmetric synthesis.

Many methods have been developed for the synthesis of imidazolines and thiazolines. The traditional process for imidazolines is the cyclization of 1,2-diamines with carboxylic acids under rigorous conditions. Unfortunately, 1,2-diamines are not easy to synthesize and the substrate scopes are quite narrow. ${ }^{14}$ BookerMilburn and Zhou reported the preparation of imidazolines from olefins, $\mathrm{N}$-chlorosaccharin (NCSacc) or NBS (cationic $\mathrm{Br}$ as initiator) and a nitrile in two steps under harsh conditions. ${ }^{15-16}$ In 2016 , Xu developed the synthesis of imidazoline via visible light catalysis from alkenes, an $\mathrm{N}$-Ts-1-aminopyridinium salt and a nitrile but the transformation was unsuccessful for non-conjugated alkenes. ${ }^{17}$ Catalyzed by Lewis acid or transition metals, the [3+2] cycloaddition of aziridines with nitriles can be performed to acquire imidazolines. ${ }^{18-24}$ For the synthesis of 2-iminothiazolidines, the [3+2] cycloaddition reaction of aziridines with isothiocayanates could also be accomplished using several catalysts or stoichiometric reagents. ${ }^{25-26}$

\section{Results and Discussion}

Although many methods have been reported for the synthesis of substituted imidazolidines and thiazolidines, it is important to find new synthetic methods from the viewpoint of synthetic diversity. In 2014, Xia group reported the ring opening reaction of aziridines by visible light photocatalysis with $\mathrm{Na}_{2} \mathrm{~S}_{2} \mathrm{O}_{8}$ as an oxidant. ${ }^{27}$ According to the oxidative quenching cycle mechanism of visible light photocatalysis of this reaction, we speculated it might be possible to carry out a [3+2] cycloaddition reaction of aziridines and nitriles under these conditions.

In order to find optimal conditions, the reaction of aziridine $\mathbf{1 a}$ and acetonitrile $\mathbf{2} \mathbf{a}$ was performed with 1.2 eq of $\mathrm{PhN}_{2} \mathrm{BF}_{4}$ in the presence of $1 \mathrm{~mol} \% \mathrm{Ru}(\mathrm{bpy})_{3} \mathrm{Cl}_{2} \cdot 6 \mathrm{H}_{2} \mathrm{O}$ under air (Table 1). The reaction mixture was illuminated with a $45 \mathrm{~W}$ white household lamp at room temperature for four hours monitored by TLC. We were delighted to find that the desired product 3a could be isolated in $73 \%$ yield (entry 1). Extending the reaction time to five, six, and seven hours respectively, did not increase the yield. The reaction proceeded very slowly and the yields were very low in the absence of light or photocatalyst (entries 2-3). The yield was improved slightly when the reaction was carried out under a $\mathrm{N}_{2}$ atmosphere (entry 4$)$. The desired product could not be obtained without $\mathrm{PhN}_{2} \mathrm{BF}_{4}$, which indicated that an oxidant was essential for the reaction (entry 5). All of the tested catalysts promoted the reaction (entries 6-11), but the best yield was obtained using $\mathrm{Ru}(\mathrm{bpy})_{3}\left(\mathrm{BF}_{4}\right)_{2}$ as a photocatalyst (entry 8). Using $\mathrm{I}_{2}, \mathrm{DDQ}, \mathrm{NBS}, \mathrm{Na}_{2} \mathrm{~S}_{2} \mathrm{O}_{8}, \mathrm{~K}_{2} \mathrm{~S}_{2} \mathrm{O}_{8}$, or Phl(OAc) $)_{2}$ as the substituted oxidant, the reaction failed (entry 12). A $64 \%$ yield of the product could be detected by GC analysis 
when $\left(\mathrm{NH}_{4}\right)_{2} \mathrm{~S}_{2} \mathrm{O}_{8}$ was used as the oxidant (entry 13). The desired product could not be obtained when $\operatorname{Ir}\left[\mathrm{dF}\left(\mathrm{CF}_{3}\right) \text { ppy }\right]_{2}(\mathrm{dtbbpy}) \mathrm{PF}_{6}\left(\mathrm{E}_{1 / 2} \mathrm{III}^{*} / \mathrm{II}=+1.21 \mathrm{~V}\right.$ vs SCE in $\left.\mathrm{CH}_{3} \mathrm{CN}\right)$ or $\mathrm{Ru}(\mathrm{bpz})_{3}\left(\mathrm{PF}_{6}\right)_{2}\left(\mathrm{E}_{1 / 2} \mathrm{III} / \mathrm{II}=+1.86 \mathrm{~V}\right.$ vs SCE in $\mathrm{CH}_{3} \mathrm{CN}$ ) were tried in the absence of $\mathrm{PhN}_{2} \mathrm{BF}_{4}$ (entries 14-15). The yields were $7 \%, 15 \%, 30 \%$ and $70 \%$ respectively when $\mathrm{PhN}_{2} \mathrm{BF}_{4}$ was used at $5 \%, 10 \%, 20 \%$ and $50 \%$. A small amount of biphenyl was detected by GC analysis.

Table 1. Optimization of the reaction conditions ${ }^{a}$

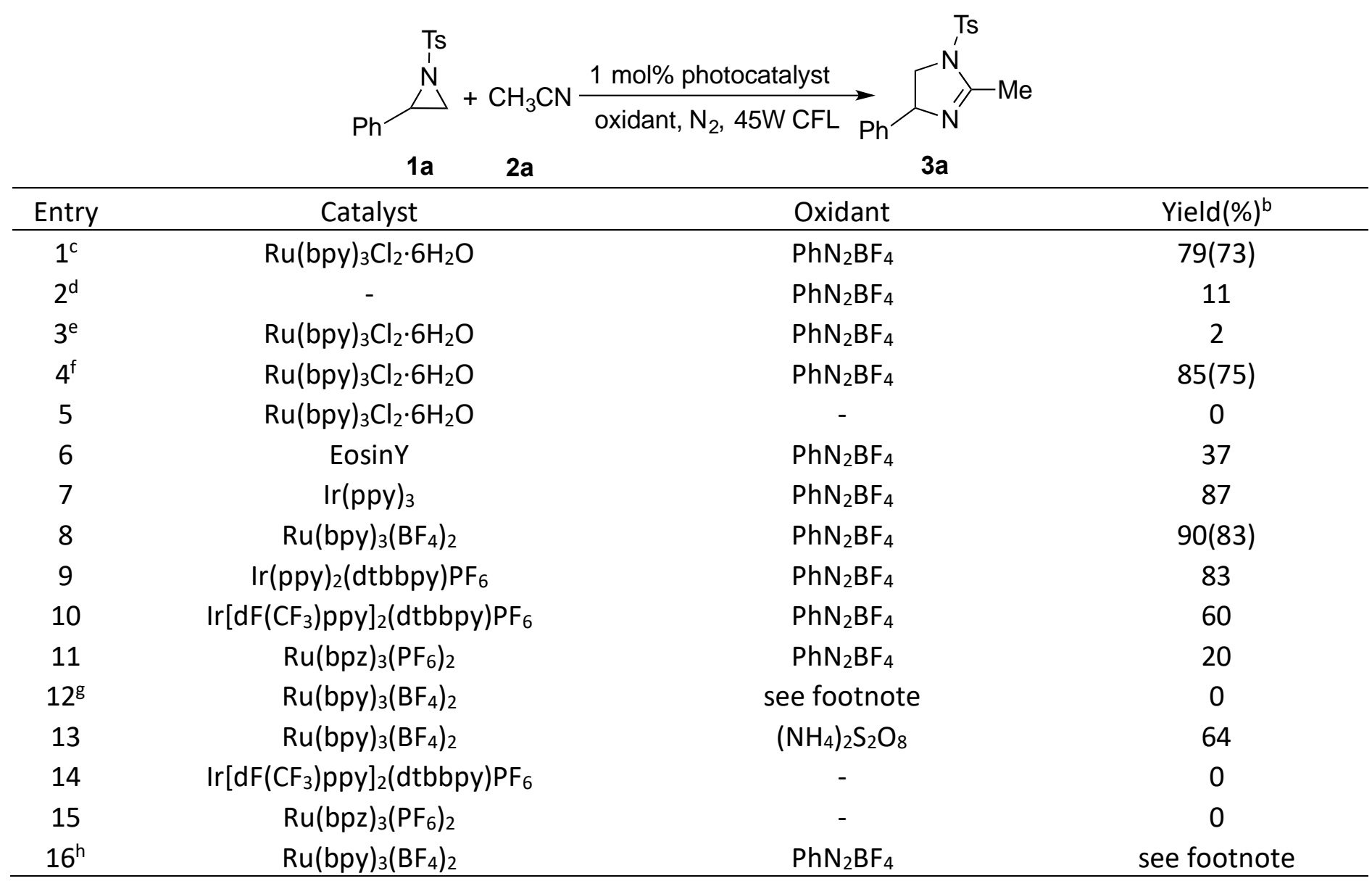

${ }^{a}$ 1a $(0.5 \mathrm{mmol}), 2 \mathrm{a}(40 \mathrm{mmol}, 2 \mathrm{~mL})$, oxidant $(0.6 \mathrm{mmol}, 1.2 \mathrm{eq})$, photocatalyst $(0.005 \mathrm{mmol}, 1 \mathrm{~mol} \%), 45 \mathrm{~W} \mathrm{CFL}$ (compact fluorescent lamp) irradiation under $\mathrm{N}_{2}$ atmosphere at r.t. for $4 \mathrm{~h}$ unless otherwise noted.

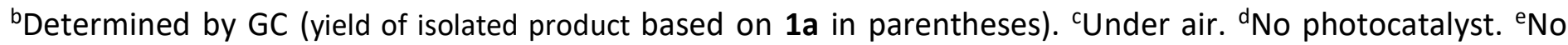
light. ${ }^{f}$ Under $\mathrm{N}_{2}$. ${ }^{8}$ Oxidants were I $\mathrm{I}_{2}, \mathrm{DDQ}, \mathrm{NBS}, \mathrm{PhI}(\mathrm{OAc})_{2}, \mathrm{Na}_{2} \mathrm{~S}_{2} \mathrm{O}_{8}, \mathrm{~K}_{2} \mathrm{~S}_{2} \mathrm{O}_{8} .{ }^{\mathrm{h}} \mathrm{CH}_{3} \mathrm{CN}(2.5 \mathrm{mmol}, 5$ eq), various solvents (2 mL), $\mathrm{CH}_{2} \mathrm{Cl}_{2}$, DCE, THF, DMSO, gave yields of $60 \%, 74 \%, 0 \%, 0 \%$ respectively.

With the optimal reaction conditions in hand, the substrate scope of the 2-alkyl(aryl)- $N$-tosylaziridine was investigated (Table 2). The substituent of the aryl ring of the 2-aryl- $N$-tosylaziridine, such as $\mathrm{F}, \mathrm{Cl}, \mathrm{Me}$ generated the cycloaddition products in good yields (3b-e). However, the bromo substituted starting material gave slightly lower yield (3f). 2-Methyl-2-phenyl- $N$-tosylaziridine, 2-octyl- $N$-tosylaziridine, $N$ tosylcyclohexanoaziridine, were also suitable for the reaction (3g-i). Next, several alkyl nitriles were examined and the corresponding products were obtained in moderate yields (3j-n). Only one nitrile group participated in the reaction when 1,4-dicyanobenzene was used as the substrate $(3 \mathrm{~m})$. 
Table 2. Cycloaddition reactions of aziridines and nitriles ${ }^{a}$

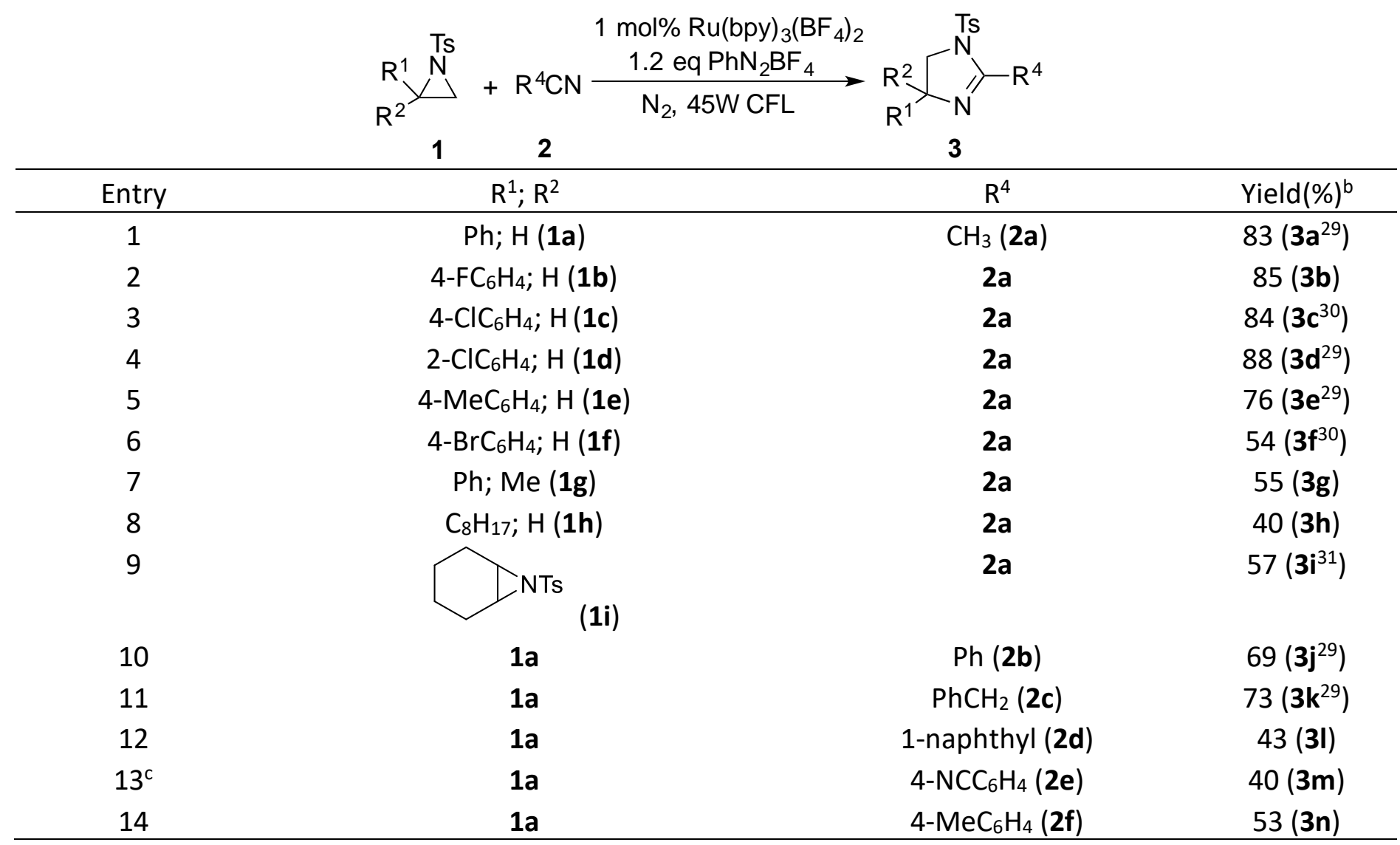

${ }^{\mathrm{a}} \mathbf{1}$ (0.5 mmol), 2 (a:40 mmol, b:20 mmol, c:17 mmol, d:14 mmol, f:17 mmol, $\left.2 \mathrm{~mL}\right), \mathrm{PhN}_{2} \mathrm{BF}_{4}(0.6 \mathrm{mmol}, 1.2 \mathrm{eq})$, $\mathrm{Ru}(\mathrm{bpy})_{3}\left(\mathrm{BF}_{4}\right)_{2}(0.005 \mathrm{mmol}, 1 \mathrm{~mol} \%), 45 \mathrm{~W} \mathrm{CFL}, \mathrm{N}_{2}$, r.t., 4 h. ${ }^{\text {bYeld }}$ of isolated product. ${ }^{\mathrm{N}}$ Nitrile $(2.5 \mathrm{mmol}, 5 \mathrm{eq})$, DCE $(2 \mathrm{~mL})$.

To further demonstrate the generality of the [3+2] cycloaddition reaction, we employed isothiocyanates 4 as dipolarophiles to synthesize 2 -iminothiazolidines. Based on the reaction of $\mathbf{1 a}$ and $\mathbf{2 a}$, the reaction conditions of $1 \mathbf{a}$ and $\mathbf{4 a}$ were screened briefly. The reaction did not proceed without light or photocatalyst. $\mathrm{A}$ lower yield was obtained under blue LED illumination perhaps because the six-membered ring dimer was formed as the by-product. ${ }^{28}$ Diphenyl was also detected by GC analysis in the reaction mixture. Screening the solvent, oxidant, etc. showed the optimal conditions were similar to that for the synthesis of $\mathbf{3 a}$ (see above).

A series of aryl or alkyl isothiocyanates were surveyed under the optimal conditions (Table 3). The expected products $\mathbf{5}$ were obtained in moderate yields. No obvious electronic effect was observed. The yield was slightly lower when 2-(2-chlorophenyl)- $N$-tosylaziridine $\mathbf{5 d}$ was used as the substrate and the steric effect may be responsible for this result. 2-Phenyl-1-(phenylsulfonyl)aziridine $\mathbf{5 q}$ was also a good candidate and afforded the corresponding product in $62 \%$ yield. 
Table 3. Cycloaddition reactions of aziridines and isothiocyanates ${ }^{a, b}$

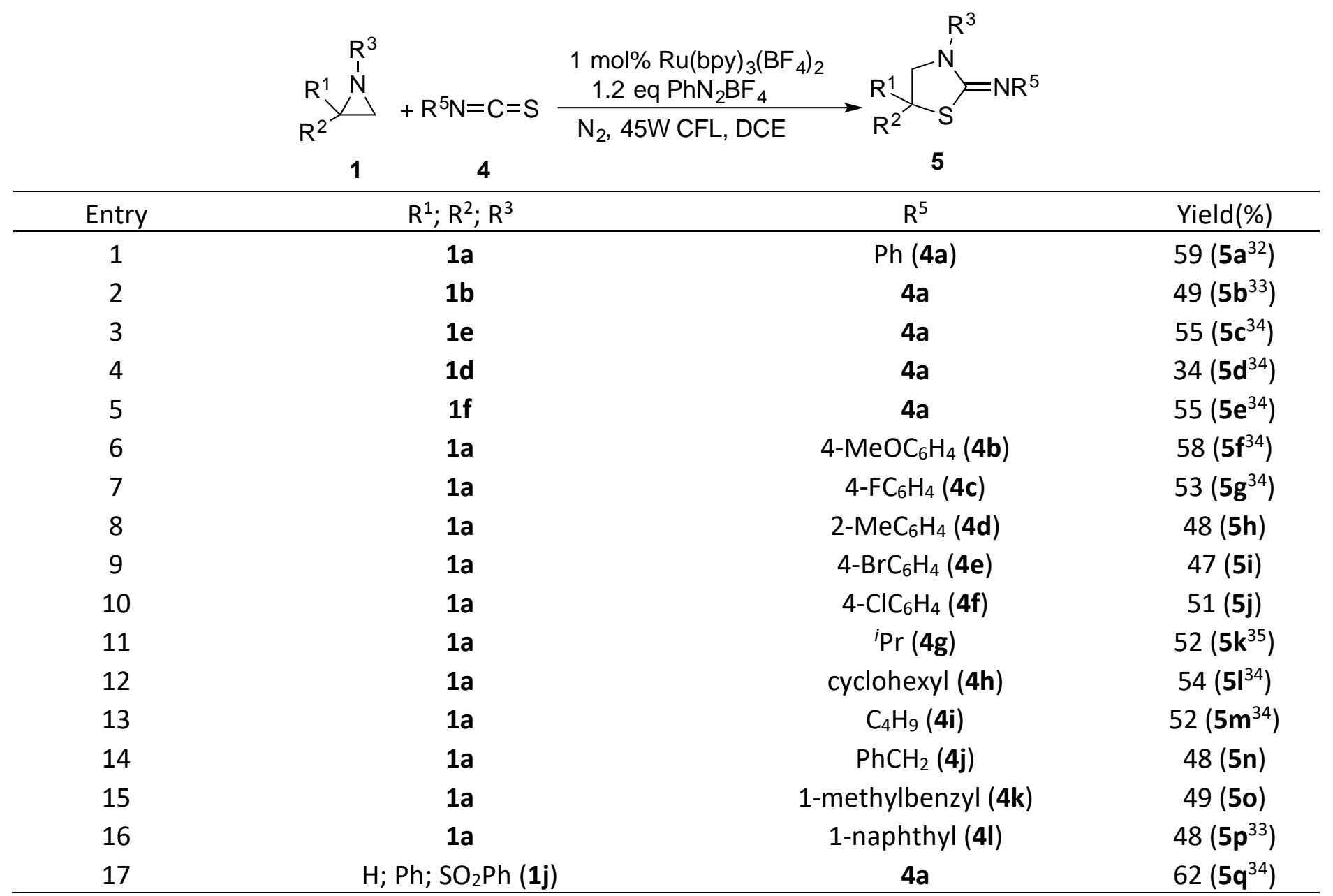

${ }^{\mathrm{a}} 1$ (0.5 mmol), 4 (1.5 mmol, $\left.3 \mathrm{eq}\right), \mathrm{PhN}_{2} \mathrm{BF}_{4}(0.6 \mathrm{mmol}, 1.2 \mathrm{eq}), \mathrm{DCE}(3 \mathrm{~mL}), \mathrm{Ru}(\mathrm{bpy})_{3}\left(\mathrm{BF}_{4}\right)_{2}(0.005 \mathrm{mmol}, 1$ mol\%), $45 \mathrm{~W}$ CFL, $\mathrm{N}_{2}$, r.t., 10 h. bYield of isolated product.

According to the literature ${ }^{16}$ and the above experimental results, a plausible reaction mechanism is proposed in Scheme 1. Firstly, under the visible light, the ground state of $\mathrm{Ru}^{2+}$ is excited to provide $\mathrm{Ru}^{{ }^{2+}} \mathrm{which}^{2+}$ is oxidized by $\mathrm{PhN}_{2} \mathrm{BF}_{4} 6$ to produce $\mathrm{Ru}^{3+}$. Thus, the diazonium salt is reduced to generate the phenyl radical 7 which produces 8 by homo-coupling. The aziridine 1 is oxidized to generate a nitrogen radical cation 9 by $\mathrm{Ru}^{3+}$ which itself is reduced to the original oxidation level, $\mathrm{Ru}^{2+}$. Intermediate $\mathbf{9}$ is attacked by the dipolarophile, nitrile $\mathbf{2}$ or isothiocyanate $\mathbf{4}$, to give the ion intermediates $\mathbf{1 0}$ or $\mathbf{1 1}$ via electron exchange with $\mathbf{1}$ or intermediate $\mathbf{7}$, intramolecular cyclization then leading to the imidazoline $\mathbf{3}$ or thiazolidine $\mathbf{5}$. 


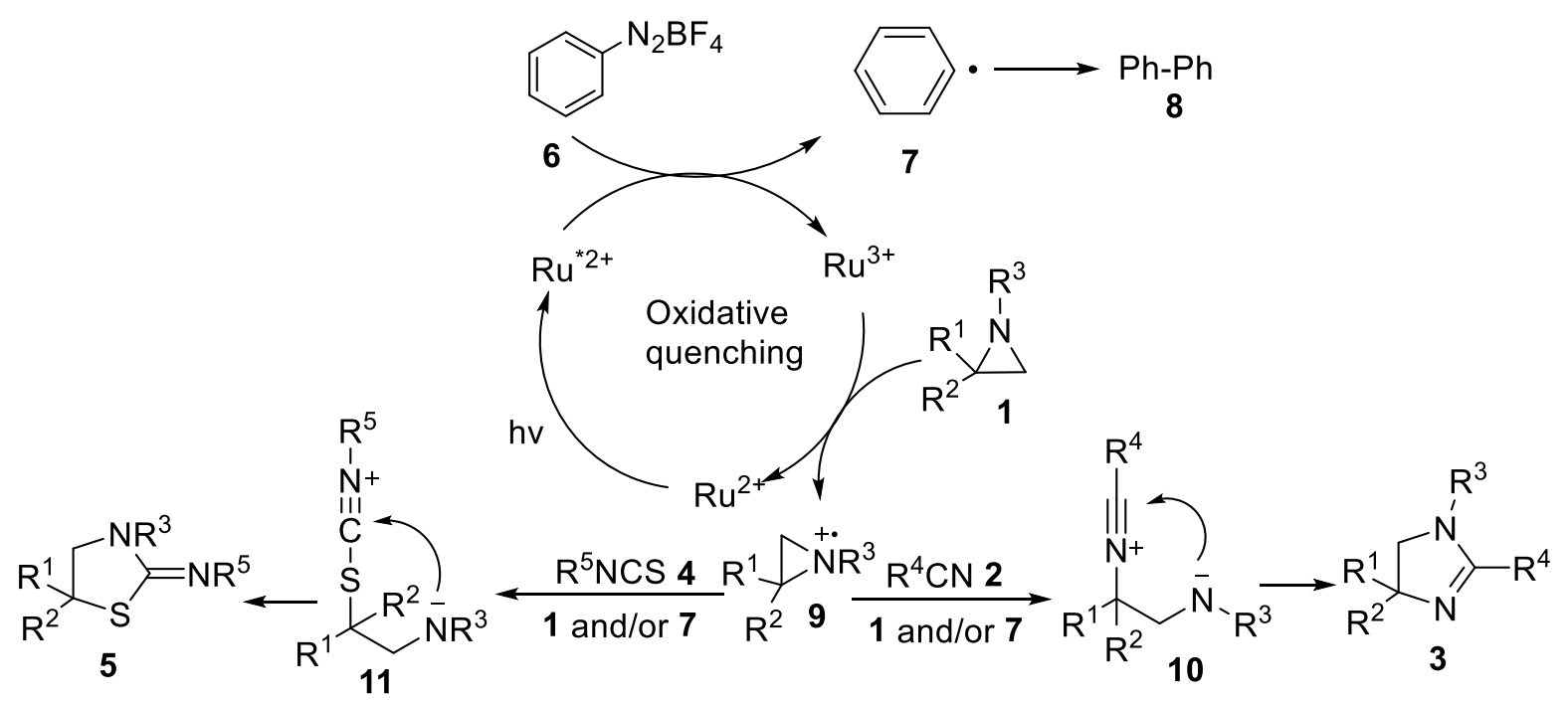

Scheme 1. A plausible reaction mechanism.

\section{Conclusions}

In summary, we have developed a [3+2] cycloaddition of aziridines with nitriles or isothiocyanates by visible light photocatalysis under simple and mild reaction conditions. The reaction is applicable to aryl and alkyl nitriles or isothiocyanates and various substituted imidazolines and iminothiazolidines can be prepared with moderate yields. Visible light as a clean energy source makes this route a good substitute for the existing synthetic protocols.

\section{Experimental Section}

General. All of the commercial reagents were used without further purification unless otherwise specified and were purchased from Aladdin, Macklin, Energy or Bide Pharmatech. All of the chemical reactions and manipulations were implemented in oven-dried glassware under an atmosphere of $\mathrm{N}_{2}$ using standard Schlenk technique. The solvents were made absolutely anhydrous and freshly distilled prior to use. Reactions were monitored by thin-layer chromatography (TLC) on GF254 Silica plates, visualizing with UV-light (254 nm) or $I_{2}$ stain. ${ }^{1} \mathrm{H} N M R$ and ${ }^{13} \mathrm{C}$ NMR spectra were recorded in ppm at $500 \mathrm{MHz}$ and $125 \mathrm{MHz}$ using $\mathrm{CDCl}_{3}$ as solvent and TMS as internal standard using a Bruker AVANCE III 500MHz Nuclear Magnetic Resonance Spectrometer. Lowresolution MS (Varian 1200 Mass Spectrum Analyzer) and HRMS (Bruker ApexIII Fourier Transform Ion Cyclotron Resonator) were obtained using ESI ionization. Elemental analyses were performed on a vario Micro cube. GC spectra were measured using an Agilent 7890B. Melting points were measured using a SGW ${ }^{\circledR} \mathrm{X}-4$.

\section{General experimental procedure for synthesis of 3a-n}

An $25 \mathrm{~mL}$ oven-dried Schlenk tube was equipped with a stirring bar, an aziridine 1 (0.5 mmol), $\mathrm{PhN}_{2} \mathrm{BF}_{4}(0.6$ $\mathrm{mmol}, 1.2 \mathrm{eq})$, and $\mathrm{Ru}(\mathrm{bpy})_{3}\left(\mathrm{BF}_{4}\right)_{2}(0.005 \mathrm{mmol}, 1 \mathrm{~mol} \%)$. The mixture was degassed by using standard Schlenk techniques with an oil pump. Then a nitrile $2 \mathbf{2 a}-\mathbf{d}, \mathbf{2 f}$ (a: $40 \mathrm{mmol}, \mathbf{b}: 20 \mathrm{mmol}, \mathbf{c}: 17 \mathrm{mmol}$, d: $14 \mathrm{mmol}, \mathbf{f}: 17 \mathrm{mmol}$, $2 \mathrm{~mL})$ or nitrile $2 \mathrm{e}(2.5 \mathrm{mmol}, 5 \mathrm{eq})$ and DCE $(2 \mathrm{~mL})$ were injected into the reaction tube. The reaction mixture was allowed to stir for $4 \mathrm{~h}$ under irradiation of $45 \mathrm{~W}$ CFL (compact fluorescent lamp) (FSL, YPZ220/45-S, 
Luminous Flux $(2580 \mathrm{Lm})$ at $\mathrm{rt}$, the distance fof the reaction vessel from the light bulb being about $5 \mathrm{~cm}$. After the reaction was completed (TLC), to the mixture was added distilled water $(10 \mathrm{~mL})$ and product extracted with $\mathrm{CH}_{2} \mathrm{Cl}_{2}(3 \times 30 \mathrm{~mL})$ in a separatory funnel. The combined organic layers were dried (anhydrous $\left.\mathrm{Na}_{2} \mathrm{SO}_{4}\right)$. Afterwards, the organic solution was concentrated under reduced pressure using a rotary evaporator and purified by column chromatography on silica gel (200-300 mesh) (PE:EtOAc $=5-10: 1)$ to give the pure product 3.

2-Methyl-4-phenyl-1-tosyl-4,5-dihydro-1H-imidazole (3a). White solid, mp 97-98 ${ }^{\circ} \mathrm{C}\left(\text { lit. } 100-102{ }^{\circ} \mathrm{C}\right)^{29} ;{ }^{1} \mathrm{H}$ NMR (500 MHz, CDCl $)$ ) 7.77-7.76 (m, 2H, Ph in Ts), 7.37 (d, J 8.0 Hz, 2H, Ph in Ts), 7.30-7.25 (m, 3H, Ph), 7.07$7.05(\mathrm{~m}, 2 \mathrm{H}, \mathrm{Ph}), 5.03-4.99\left(\mathrm{~m}, 1 \mathrm{H}, \mathrm{CH}_{2}\right), 4.21-4.17\left(\mathrm{~m}, 1 \mathrm{H}, \mathrm{CH}_{2}\right), 3.65(\mathrm{dd}, J 9.8,7.9 \mathrm{~Hz}, 1 \mathrm{H}, \mathrm{CHN}=), 2.48(\mathrm{~s}, 3 \mathrm{H}$, Me in Ts), 2.42, 2.41 (ss, 3H, 2-Me). ${ }^{13} \mathrm{C}$ NMR $\left(125 \mathrm{MHz}, \mathrm{CDCl}_{3}\right) \delta 156.5,144.8,141.5,135.2,130.1,128.7$, 127.7, 127.3, 126.4, 66.6, 55.5, 21.6, 16.8. MS (ESI): $\left([\mathrm{M}+\mathrm{H}]^{+}\right)$315.1.

2-Methyl-4-(4-fluorophenyl)-1-tosyl-4,5-dihydro-1H-imidazole (3b). Colorless gum; $\left.{ }^{1} \mathrm{H} \mathrm{NMR} \mathrm{(500} \mathrm{MHz}_{\mathrm{C}} \mathrm{CDCl}_{3}\right)$ $\delta 7.76(\mathrm{~d}, J 8.3 \mathrm{~Hz}, 2 \mathrm{H}, \mathrm{Ph}$ in Ts), 7.37 (d, J $8.2 \mathrm{~Hz}, 2 \mathrm{H}, \mathrm{Ph}$ in Ts), 7.05-7.01 (m, 2H, Ph), 6.98-6.95 (m, 2H, Ph), 5.01-4.98 (m, $\left.1 \mathrm{H}, \mathrm{CH}_{2}\right), 4.20-4.16\left(\mathrm{~m}, 1 \mathrm{H}, \mathrm{CH}_{2}\right), 3.61(\mathrm{dd}, J 9.9,7.8 \mathrm{~Hz}, 1 \mathrm{H}, \mathrm{CHN}=), 2.48(\mathrm{~s}, 3 \mathrm{H}, \mathrm{Me}$ in Ts), 2.421, 2.418 (ss, 3H, 2-Me). ${ }^{13} \mathrm{C} \mathrm{NMR}\left(125 \mathrm{MHz}, \mathrm{CDCl}_{3}\right) \delta 163.2,161.3,157.1,145.0,137.3,135.1,130.2,129.8,128.0$, 128.1, 127.3, 127.1, 115.7, 115.5, 65.7, 55.6, 21.6, 16.8. MS (ESI): ([M+H $\left.]^{+}\right)$333.1. HRMS (ESI) calculated for $\mathrm{C}_{17} \mathrm{H}_{19} \mathrm{FN}_{2} \mathrm{O}_{2} \mathrm{~S}\left([\mathrm{M}+\mathrm{H}]^{+}\right)$333.0995, found 333.1068. El. Anal. calculated for $\mathrm{C}_{17} \mathrm{H}_{18} \mathrm{FN}_{2} \mathrm{O}_{2} \mathrm{~S}: \mathrm{C}, 61.43 ; \mathrm{H}, 5.16 ; \mathrm{N}$, 8.43; found C, 61.29; H, 5.45; N, 8.52.

2-Methyl-4-(4-chlorophenyl)-1-tosyl-4,5-dihydro-1H-imidazole (3c). White solid, mp 173-174 ${ }^{\circ} \mathrm{C}$ (lit. $170-172$ $\left.{ }^{\circ} \mathrm{C}\right){ }^{30} ;{ }^{1} \mathrm{H}$ NMR $\left(500 \mathrm{MHz}, \mathrm{CDCl}_{3}\right) \delta 7.75$ (d, J $8.3 \mathrm{~Hz}, 2 \mathrm{H}, \mathrm{Ph}$ in Ts), 7.36 (d, J $8.1 \mathrm{~Hz}, 2 \mathrm{H}, \mathrm{Ph}$ in Ts), 7.26-7.23 (m, $2 \mathrm{H}, \mathrm{Ph}), 7.01-6.99(\mathrm{~m}, 2 \mathrm{H}, \mathrm{Ph}), 5.01-4.96\left(\mathrm{~m}, 1 \mathrm{H}, \mathrm{CH}_{2}\right), 4.20-4.16\left(\mathrm{~m}, 1 \mathrm{H}, \mathrm{CH}_{2}\right), 3.59(\mathrm{dd}, J 9.9,7.8 \mathrm{~Hz}, 1 \mathrm{H}$, $\mathrm{CHN}=), 2.48$ (s, 3H, Me in Ts), 2.413, 2.412 (ss, 3H, 2-Me,). ${ }^{13} \mathrm{C} \mathrm{NMR}\left(125 \mathrm{MHz}^{\mathrm{CDCl}}{ }_{3}\right) \delta$ 157.0, 144.9, 140.1, $135.1,133.5,130.2,128.8,127.8,127.2,65.8,55.4,21.6,16.8 . \mathrm{MS}(\mathrm{ESI}):\left([\mathrm{M}+\mathrm{H}]^{+}\right) 349.1$.

2-Methyl-4-(2-chlorophenyl)-1-tosyl-4,5-dihydro-1H-imidazole (3d). White solid, mp $133-135{ }^{\circ} \mathrm{C}(\mathrm{EtOAC}-\mathrm{PE})^{29}$; ${ }^{1} \mathrm{H}$ NMR $\left(500 \mathrm{MHz} \mathrm{CDCl}_{3}\right) \delta 7.74$ (d, J $8.3 \mathrm{~Hz}, 2 \mathrm{H}, \mathrm{Ph}$ in Ts), 7.36-7.33 (m, 3H, Ph and Ph in Ts), 7.23-7.16 (m, 2H, $\mathrm{Ph})$, 7.12-7.10 (m, 1H, Ph), 5.35-5.32 (m, 1H, $\left.\mathrm{CH}_{2}\right), 4.34-4.30\left(\mathrm{~m}, 1 \mathrm{H}, \mathrm{CH}_{2}\right), 3.55(\mathrm{dd}, J 10.0,7.7 \mathrm{~Hz}, 1 \mathrm{H}, \mathrm{CHN}=)$, 2.452, 2.448 (ss, 6H, 2-Me). ${ }^{13} \mathrm{C}$ NMR $\left(125 \mathrm{MHz} \mathrm{CDCl}_{3}\right) \delta 157.7,144.8,139.5,135.3,132.3,130.1,129.4,128.7$, 127.5, 127.2, 127.1, 63.7, 54.8, 21.6, 16.9. MS (ESI): ([M+H] $\left.]^{+}\right) 349.1$.

2-Methyl-4-(p-tolyl)-1-tosyl-4,5-dihydro-1H-imidazole (3e). Colorless liquid ${ }^{29} ;{ }^{1} \mathrm{H} N M R\left(500 \mathrm{MHz}^{\mathrm{C}} \mathrm{CDCl}\right) \delta$ 7.76 (d, J $8.3 \mathrm{~Hz}, 2 \mathrm{H}$, Ph in Ts), 7.36 (d, J $8.1 \mathrm{~Hz}, 2 \mathrm{H}, \mathrm{Ph}$ in Ts), 7.08 (d, J 7.9 Hz, 2H, Ph), 6.95 (d, J 8.0 Hz, 2H, Ph), 4.98-4.95 (m, $\left.1 \mathrm{H}, \mathrm{CH}_{2}\right)$, 4.19-4.15 (m, $\left.1 \mathrm{H}, \mathrm{CH}_{2}\right), 3.62(\mathrm{dd}, J$ 9.7, $8.0 \mathrm{~Hz}, 1 \mathrm{H}, \mathrm{CHN}=$ ), 2.48 (s, 3H, Me in Ts), 2.401, 2.398 (ss, 3H, 2-Me), 2.32 (s, 3H, $\mathrm{CH}_{3}$ in tolyl). ${ }^{13} \mathrm{C} \mathrm{NMR}\left(125 \mathrm{MHz}, \mathrm{CDCl}_{3}\right) \delta 156.2,144.7,138.6,137.3,135.2$, 130.1, 129.3, 127.2, 126.3, 66.4, 55.5, 21.6, 21.1, 16.8. MS (ESI): $\left([\mathrm{M}+\mathrm{H}]^{+}\right) 329.1$.

2-Methyl-4-(4-bromophenyl)-1-tosyl-4,5-dihydro-1H-imidazole (3f). Colorless liquid ${ }^{30} ;{ }^{1} \mathrm{H} \mathrm{NMR}(500 \mathrm{MHz}$, $\left.\mathrm{CDCl}_{3}\right) \delta 7.74(\mathrm{~d}, J 8.3 \mathrm{~Hz}, 2 \mathrm{H}, \mathrm{Ph}), 7.41-7.38$ (m, 2H, Ph in Ts), 7.36 (d, J 8.2 Hz, 2H, Ph in Ts ), 6.96-6.93 (m, 2H, $\mathrm{Ph})$, 4.99-4.95 (m, 1H, CH $\left.\mathrm{CH}_{2}\right)$ 4.21-4.16 (m, 1H, $\left.\mathrm{CH}_{2}\right), 3.58(\mathrm{dd}, J$ 9.9, $7.8 \mathrm{~Hz}, 1 \mathrm{H}, \mathrm{CHN}=), 2.48(\mathrm{~s}, 3 \mathrm{H}, \mathrm{Me}$ in Ts), 2.412, 2.409 (ss, 3H, 2-Me). ${ }^{13} \mathrm{C}$ NMR $\left(125 \mathrm{MHz} \mathrm{CDCl}_{3}\right) \delta 157.0,145.0,140.6,135.1,131.8,130.2,128.1,127.2$, 121.6, 65.9, 55.4, 21.6, 16.8. MS (ESI): $\left([\mathrm{M}+\mathrm{H}]^{+}\right)$395.0.

2,4-Dimethyl-4-phenyl-1-tosyl-4,5-dihydro-1H-imidazole (3g). White solid, $\mathrm{mp} 82-83^{\circ} \mathrm{C}$ (EtOAC-PE); ${ }^{1} \mathrm{H} N M R$ $\left(500 \mathrm{MHz}, \mathrm{CDCl}_{3}\right) \delta 7.72$ (d, J $8.3 \mathrm{~Hz}, 2 \mathrm{H}, \mathrm{Ph}$ in Ts), 7.32-7.22 (m, 7H, Ph and Ph in Ts), $3.93\left(\mathrm{~d}, J 9.6 \mathrm{~Hz}, 1 \mathrm{H}, \mathrm{CH}_{2}\right)$, 3.81 (d, J $9.6 \mathrm{~Hz}, 1 \mathrm{H}, \mathrm{CH}_{2}$ ), 2.44 (s, 3H, Me in Ts), 2.39 (s, 3H, 2-Me), 1.47 (s, 3H, 4-Me). ${ }^{13} \mathrm{C} \mathrm{NMR}(125 \mathrm{MHz}$, $\left.\mathrm{CDCl}_{3}\right) \delta 154.2,146.3,144.6,135.4,130.0,128.5,127.1,126.9,125.0,69.6,61.1,29.6,21.6,16.9 . \mathrm{MS}$ (ESI): $\left([\mathrm{M}+\mathrm{H}]^{+}\right)$329.1. HRMS (ESI) calculated for $\mathrm{C}_{18} \mathrm{H}_{21} \mathrm{~N}_{2} \mathrm{O}_{2} \mathrm{~S}\left([\mathrm{M}+\mathrm{H}]^{+}\right)$: 329.1318, found: 329.1245. El. Anal. calculated for $\mathrm{C}_{18} \mathrm{H}_{20} \mathrm{~N}_{2} \mathrm{O}_{2} \mathrm{~S}$ : C, 65.83; $\mathrm{H}, 6.14 ; \mathrm{N}, 8.53$; found $\mathrm{C}, 65.72 ; \mathrm{H}, 6.37 ; \mathrm{N}, 8.69$. 
2-Methyl-4-n-octyl-1-tosyl-4,5-dihydro-1H-imidazole (3h). Viscous oil; ${ }^{1} \mathrm{H}$ NMR (500 MHz, $\left.\mathrm{CDCl}_{3}\right) \delta 7.75$ (d, J $8.3 \mathrm{~Hz}, 2 \mathrm{H}, \mathrm{Ph}), 7.36(\mathrm{~d}, J 8.1 \mathrm{~Hz}, 2 \mathrm{H}, \mathrm{Ph}), 3.86-3.81\left(\mathrm{~m}, 2 \mathrm{H}, \mathrm{CH}_{2} \mathrm{~N}\right), 3.34(\mathrm{dd}, J$ 8.2, $6.3 \mathrm{~Hz}, 1 \mathrm{H}, \mathrm{CHN}=), 2.46(\mathrm{~s}, 3 \mathrm{H}$, Me in Ts), 2.29 (s, 3H, 2-Me), 1.29-1.23 (m, 14H, 7- $\left.\mathrm{CH}_{2}\right), 0.88$ (t, J 7.0 Hz, 3H, CH in n-octyl). ${ }^{13} \mathrm{C} \mathrm{NMR} \mathrm{(125}$ $\mathrm{MHz}_{\mathrm{CDCl}}$ ) $\delta 155.0,144.6,135.4,130.0,127.2,63.6,53.2,35.8,31.8,29.5,29.4,29.2,25.6,22.6,21.6,16.8$, 14.1; $\mathrm{MS}$ (ESI): $\left([\mathrm{M}+\mathrm{H}]^{+}\right)$351.2. HRMS (ESI) calculated for $\mathrm{C}_{19} \mathrm{H}_{31} \mathrm{~N}_{2} \mathrm{O}_{2} \mathrm{~S}\left([\mathrm{M}+\mathrm{H}]^{+}\right)$351.2101, found 351.2028. El. Anal. calculated for $\mathrm{C}_{19} \mathrm{H}_{30} \mathrm{~N}_{2} \mathrm{O}_{2} \mathrm{~S}: \mathrm{C}, 65.11 ; \mathrm{H}, 8.63 ; \mathrm{N}, 7.99$; found $\mathrm{C}, 65.32 ; \mathrm{H}, 8.59 ; \mathrm{N}, 8.21$.

2-Methyl-1-tosyl-3a,4,5,6,7,7a-hexahydro-1H-benzimidazole (3i). White solid, $\mathrm{mp} 112-113{ }^{\circ} \mathrm{C}$ (lit. $115-117$ $\left.{ }^{\circ} \mathrm{C}\right){ }^{31} ;{ }^{1} \mathrm{H}$ NMR $\left(500 \mathrm{MHz}, \mathrm{CDCl}_{3}\right) \delta 7.71(\mathrm{~d}, J 8.3 \mathrm{~Hz}, 2 \mathrm{H}, \mathrm{Ph}), 7.36(\mathrm{~d}, J 8.2 \mathrm{~Hz}, 2 \mathrm{H}, \mathrm{Ph}),, 3.13-3.07(\mathrm{~m}, 1 \mathrm{H}$, CHNTs), 2.88-2.82 (m, 1H, $\left.\mathrm{CH}_{2}\right), 2.57-2.54\left(\mathrm{~m}, 1 \mathrm{H}, \mathrm{CH}_{2}\right), 2.46$ (s, 3H, Me in Ts), 2.32, 2.31 (ss, 3H, 2-Me), 2.26$2.23\left(\mathrm{~m}, 1 \mathrm{H}, \mathrm{CH}_{2}\right), 1.88-1.78\left(\mathrm{~m}, 2 \mathrm{H}, \mathrm{CH}_{2}\right), 1.68-1.60(\mathrm{~m}, 1 \mathrm{H}, \mathrm{CHN}=), 1.37-1.22\left(\mathrm{~m}, 3 \mathrm{H}, \mathrm{CH}_{2}\right) .{ }^{13} \mathrm{C} \mathrm{NMR}(125 \mathrm{MHz}$, $\left.\mathrm{CDCl}_{3}\right) \delta 158.2,144.6,135.0,130.0,127.4,70.2,69.3,30.56,30.55,25.0,24.6,21.6,17.9 . \mathrm{MS}(\mathrm{ESI}):\left([\mathrm{M}+\mathrm{H}]^{+}\right)$ 293.1.

2,4-Diphenyl-1-tosyl-4,5-dihydro-1H-imidazole (3j). White solid, mp $164-165{ }^{\circ} \mathrm{C}$ (EtOAc-PE) ${ }^{29} ;{ }^{1} \mathrm{H} N M R(500$ $\left.\mathrm{MHz}, \mathrm{CDCl}_{3}\right) \delta$ 7.80-7.78 (m, 2H, 2-Ph), 7.56-7.53 (m, 1H, Ph in Ts), 7.47-7.41 (m, 4H, Ph in Ts and 2-Ph), 7.25$7.21(\mathrm{~m}, 5 \mathrm{H}, \mathrm{Ph}$ in Ts and 4-Ph), 6.70-6.98 (m, 2H, 4-Ph), 5.03-4.99 (m, 1H, CHN=), 4.48-4.43 (m, 1H, CH $), 3.90-$ $3.86\left(\mathrm{~m}, 1 \mathrm{H}, \mathrm{CH}_{2}\right), 2.44$ (s, 3H, Me). $\left.{ }^{13} \mathrm{C} \mathrm{NMR} \mathrm{(125} \mathrm{MHz,} \mathrm{CDCl} 3\right) \delta 159.9,144.7,141.5,134.5,131.2,130.1$, $129.9,129.8,128.6,127.7,127.6,127.4,126.4,67.8,56.9,21.6 . \mathrm{MS}(\mathrm{ESI}):\left([\mathrm{M}+\mathrm{H}]^{+}\right) 377.1$.

2-Benzyl-4-phenyl-1-tosyl-4,5-dihydro-1 $\mathbf{H}$-imidazole (3k). Colorless liquid ${ }^{29} ;{ }^{1} \mathrm{H} \mathrm{NMR}\left(500 \mathrm{MHz}, \mathrm{CDCl}_{3}\right) \delta 7.43-$ $7.40(\mathrm{~m}, 4 \mathrm{H}, \mathrm{Ph}$ in Ts), 7.38-7.25 (m, 6H, 4-Ph and 2-Ph), 7.21-7.18 (m, 2H, 2-Ph), 7.04-7.7.03 (m, 2H, 2-Ph), 5.08-5.05 (m, $1 \mathrm{H}, \mathrm{CHN}=)$, 4.20-4.14 (m, 3H, $\mathrm{CH}_{2}$ and $\mathrm{CH}_{2}$ in benzyl), 3.62-3.59 (m, $1 \mathrm{H}, \mathrm{CH}_{2}$ in benzyl), $2.42(\mathrm{~s}, 3 \mathrm{H}$, Me). ${ }^{13} \mathrm{C} N M R\left(125 \mathrm{MHz}, \mathrm{CDCl}_{3}\right) \delta 158.3,144.5,141.7,135.4,134.9,129.9,129.4,128.7,128.5,127.6,127.3$, 127.0, 126.4, 66.9, 55.5, 35.7, 21.5. MS (ESI): $\left([\mathrm{M}+\mathrm{H}]^{+}\right) 391.1$.

2-(1-Naphthalenyl)-4-phenyl-1-tosyl-4,5-dihydro-1H-imidazole (3l). White solid, mp 152-153 ${ }^{\circ} \mathrm{C}$ (EtOAC-PE); ${ }^{1} \mathrm{H}$ NMR $\left(500 \mathrm{MHz}, \mathrm{CDCl}_{3}\right) \delta 7.98(\mathrm{~d}, J 8.2 \mathrm{~Hz}, 1 \mathrm{H}), 7.91(\mathrm{~d}, J 8.4 \mathrm{~Hz}, 1 \mathrm{H}), 7.86(\mathrm{~d}, J 8.1 \mathrm{~Hz}, 1 \mathrm{H}), 7.73(\mathrm{~d}, J 6.8 \mathrm{~Hz}$, $1 \mathrm{H})$, 7.56-7.52 (m, $1 \mathrm{H}), 7.51-7.47(\mathrm{~m}, 1 \mathrm{H}), 7.43-7.40(\mathrm{~m}, 1 \mathrm{H}), 7.37-7.23(\mathrm{~m}, 7 \mathrm{H}), 7.02(\mathrm{~d}, J 8.1 \mathrm{~Hz}, 2 \mathrm{H}, \mathrm{Ph}), 5.36$ (dd, J 9.9, 8.8 Hz, 1H, CH 2$), 4.62-4.58\left(\mathrm{~m}, 1 \mathrm{H}, \mathrm{CH}_{2}\right), 4.05(\mathrm{dd}, J 10.5,8.4 \mathrm{~Hz}, 1 \mathrm{H}, \mathrm{CHN}=), 2.34\left(\mathrm{~s}, 3 \mathrm{H}, \mathrm{Me}\right.$ in Ts). ${ }^{13} \mathrm{C}$ NMR $\left(125 \mathrm{MHz}_{1} \mathrm{CDCl}_{3}\right) \delta 157.7,144.5,141.6,134.6,133.2,131.4,130.8,129.4,128.8,128.3,127.7,127.6$, $127.3,126.7,126.5,125.9,125.2,124.4,68.2,55.8,21.5 . \mathrm{MS}(\mathrm{ESI}):\left([\mathrm{M}+\mathrm{H}]^{+}\right)$427.2; HRMS (ESI) calculated for $\mathrm{C}_{26} \mathrm{H}_{23} \mathrm{~N}_{2} \mathrm{O}_{2} \mathrm{~S}\left([\mathrm{M}+\mathrm{H}]^{+}\right)$427.1494, found 427.1475. El. Anal. calculated for $\mathrm{C}_{26} \mathrm{H}_{22} \mathrm{~N}_{2} \mathrm{O}_{2} \mathrm{~S}: \mathrm{C}, 73.21 ; \mathrm{H}, 5.20 ; \mathrm{N}$, 6.57; found $\mathrm{C}, 73.11 ; \mathrm{H}, 5.36 ; \mathrm{N}, 6.79$.

2-(p-Benzonitrile)-4-phenyl-1-tosyl-4,5-dihydro-1H-imidazole (3m). White solid, $\mathrm{mp} 151-153{ }^{\circ} \mathrm{C}(\mathrm{EtOAC}-\mathrm{PE})$; ${ }^{1} \mathrm{H}$ NMR $\left(500 \mathrm{MHz}, \mathrm{CDCl}_{3}\right) \delta 7.92$ (d, J $\left.8.3 \mathrm{~Hz}, 2 \mathrm{H}, 2-\mathrm{Ph}\right), 7.75$ (d, J $8.3 \mathrm{~Hz}, 2 \mathrm{H}, \mathrm{Ph}$ in Ts), $7.42(\mathrm{~d}, J 8.2 \mathrm{~Hz}, 2 \mathrm{H}, \mathrm{Ph}$ in Ts), 7.27-7.23 (m, 5H, 4-Ph), 6.97-6.95 (m, 2H, 2-Ph), 5.07-5.03 (m, 1H, $\left.\mathrm{CH}_{2}\right), 4.47-4.43\left(\mathrm{~m}, 1 \mathrm{H}, \mathrm{CH}_{2}\right), 3.88$ (dd, J 11.4, 8.1 Hz, 1H, CHN=), 2.46 (s, 3H, Me). $\left.{ }^{13} \mathrm{C} \mathrm{NMR} \mathrm{(125} \mathrm{MHz,} \mathrm{CDCl}\right)_{3} \delta 158.4145 .2,140.9,134.7,134.1$, 132.8, 131.6, 130.5, 130.0, 128.7, 127.7, 127.6, 126.3, 118.2, 114.7, 68.4, 56.8, 21.6. MS (ESI): $\left([\mathrm{M}+\mathrm{H}]^{+}\right)$402.1; HRMS (ESI) calculated for $\mathrm{C}_{23} \mathrm{H}_{20} \mathrm{~N}_{3} \mathrm{O}_{2} \mathrm{~S}\left([\mathrm{M}+\mathrm{H}]^{+}\right) 402.1259$, found 402.1271. El. Anal. calculated for $\mathrm{C}_{23} \mathrm{H}_{19} \mathrm{~N}_{3} \mathrm{O}_{2} \mathrm{~S}$ : C, 68.81; H, 4.77; N, 10.47; found C, 68.92; H, 4.78; N, 10.32.

4-Phenyl-2-(p-tolyl)-1-tosyl-4,5-dihydro-1H-imidazole (3n). Colorless liquid; ${ }^{1} \mathrm{H} \mathrm{NMR}\left(500 \mathrm{MHz}, \mathrm{CDCl}_{3}\right) \delta 7.71$ (d, J $8.1 \mathrm{~Hz}, 2 \mathrm{H}, \mathrm{Ph}$ in Ts), 7.43 (d, J $8.3 \mathrm{~Hz}, 2 \mathrm{H}, \mathrm{Ph}$ in Ts), 7.28-7.26 (m, 2H, 2-Ph), 7.24-7.20 (m, 5H, 4-Ph), 6.98$6.96(\mathrm{~m}, 2 \mathrm{H}, 2-\mathrm{Ph}), 4.96$ (dd, J 9.5, $8.4 \mathrm{~Hz}, 1 \mathrm{H}, \mathrm{CHN}=), 4.43\left(\mathrm{dd}, J 11.4,10.0 \mathrm{~Hz}, 1 \mathrm{H}, \mathrm{CH}_{2}\right), 3.85(\mathrm{dd}, J 11.5,8.1 \mathrm{~Hz}$, $1 \mathrm{H}, \mathrm{CH}_{2}$ ), 2.45(s, 3H, Me in Ts), 2.44 (s, 3H, Me in tolyl). ${ }^{13} \mathrm{C} \mathrm{NMR}\left(125 \mathrm{MHz}, \mathrm{CDCl}_{3}\right) \delta 160.0,144.6,141.7,141.6$, 134.5, 129.9, 129.8, 128.9, 128.54, 128.45, 127.6, 127.4, 127.3, 127.2, 127.0, 126.3, 67.8, 56.9, 21.63, 21.59. MS (ESI): $\left([\mathrm{M}+\mathrm{H}]^{+}\right)$391.2; HRMS (ESI) calculated for $\mathrm{C}_{23} \mathrm{H}_{23} \mathrm{~N}_{2} \mathrm{O}_{2} \mathrm{~S}\left([\mathrm{M}+\mathrm{H}]^{+}\right)$391.1492, found 391.1475. El. Anal. calculated for $\mathrm{C}_{23} \mathrm{H}_{22} \mathrm{~N}_{2} \mathrm{O}_{2} \mathrm{~S}$ : C, 70.74; $\mathrm{H}, 5.68 ; \mathrm{N}, 7.17$; found $\mathrm{C}, 70.58 ; \mathrm{H}, 5.77 ; \mathrm{N}, 7.33$. 


\section{General experimental procedure for synthesis of $5 a-q$}

An $25 \mathrm{~mL}$ oven-dried Schlenk tube was equipped with a stirring bar, an aziridine 1 (0.5 mmol), $\mathrm{PhN}_{2} \mathrm{BF}_{4}(0.6$ $\mathrm{mmol}, 1.2 \mathrm{eq})$, and $\mathrm{Ru}(\mathrm{bpy})_{3}\left(\mathrm{BF}_{4}\right)_{2}(0.005 \mathrm{mmol}, 1 \mathrm{~mol} \%)$. The mixture was degassed by using standard Schlenk techniques with the oil pump. Then isothiocyanate $4(1.5 \mathrm{mmol}, 3 \mathrm{eq})$, and DCE $(3 \mathrm{~mL})$ were injected into the reaction tube. The reaction mixture was allowed to stir for $10 \mathrm{~h}$ under irradiation of $45 \mathrm{~W}$ CFL (FSL, YPZ220/45$\mathrm{S}$, Luminous Flux $(2580 \mathrm{Lm})$ at $\mathrm{rt}$, the distance from the light bulb to the reaction vessel being about $5 \mathrm{~cm}$. After the reaction was complete $(T L C)$, the tot he mixture was added distilled water $(10 \mathrm{~mL})$ and product extracted with $\mathrm{CH}_{2} \mathrm{Cl}_{2}(3 \times 30 \mathrm{ml})$ in separatory funnel. The combined the organic layers were dried $\left(\mathrm{Na}_{2} \mathrm{SO}_{4}\right)$, the organic solution was concentrated under reduced pressure using a rotary evaporator and the residue purified by column chromatography on silica gel (200-300 mesh) (PE:EtOAc = 5-10:1) to give the pure product 5.

$\mathbf{N}, 5$-Diphenyl-3-tosylthiazolidin-2-imine (5a). White solid, mp $129-131{ }^{\circ} \mathrm{C}$ (lit. $132-133{ }^{\circ} \mathrm{C}$ ) ${ }^{32} ;{ }^{1} \mathrm{H}$ NMR $(500$ $\left.\mathrm{MHz}, \mathrm{CDCl}_{3}\right) \delta 8.00(\mathrm{~d}, J 8.4 \mathrm{~Hz}, 2 \mathrm{H}, \mathrm{Ph}$ in Ts$), 7.38-7.33(\mathrm{~m}, 7 \mathrm{H}, \mathrm{Ph}$ in Ts and 5-Ph), 7.28-7.26 (m, 2H, $\mathrm{PhN}=)$, $7.08(\mathrm{t}, J 7.4 \mathrm{~Hz}, 1 \mathrm{H}, \mathrm{PhN}=), 6.81-6.79(\mathrm{~m}, 2 \mathrm{H}, \mathrm{PhN}=), 4.82\left(\mathrm{dd}, J 8.6,6.5 \mathrm{~Hz}, 1 \mathrm{H}, \mathrm{CH}_{2}\right), 4.62(\mathrm{dd}, J 10.4,6.5 \mathrm{~Hz}, 1 \mathrm{H}$, $\mathrm{CH}_{2}$ ), 4.07 (dd, J 10.4, $\left.8.6 \mathrm{~Hz}, 1 \mathrm{H}, \mathrm{CHS}\right), 2.50$ (s, 3H, -Me). ${ }^{13} \mathrm{C}$ NMR (125 MHz, CDCl $) \delta 152.1,150.1,144.9$, $136.5,134.7,129.24,129.20,129.05,128.97,128.8,127.5,124.3,120.8,56.8,47.0,21.7 . \mathrm{MS}(\mathrm{ESI}):\left([\mathrm{M}+\mathrm{H}]^{+}\right)$ 409.1.

N-Phenyl-5-(4-fluorophenyl)-3-tosylthiazolidin-2-imine (5b). Viscous liquid ${ }^{33} ;{ }^{1} \mathrm{H} N M R\left(500 \mathrm{MHz} \mathrm{CDCl}_{3}\right) \delta 8.00$ (d, J $8.3 \mathrm{~Hz}, 2 \mathrm{H}, \mathrm{Ph}$ in Ts), 7.38-7.33 (m,4H, Ph in Ts and 5-Ph), 7.29-7.26 (m, 2H, PhN=), 7.10-7.07 (m, 1H, $\mathrm{PhN}=$ ), 7.05-7.02 (m, 2H, 5-Ph), 6.80-6.79 (m, 2H, PhN=), 4.79 (dd, J 8.1, $\left.6.5 \mathrm{~Hz}, 1 \mathrm{H}, \mathrm{CH}_{2}\right), 4.58$ (dd, J 10.5, 6.4

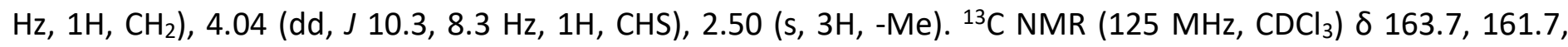
$151.8,150.0,145.0,134.6,132.5,129.3,129.24,129.21,129.15,129.0,124.4,120.7,116.1,115.9,56.8,46.2$, 21.7. $\mathrm{MS}(\mathrm{ESI}):\left([\mathrm{M}+\mathrm{H}]^{+}\right)$427.1; HRMS (ESI) calculated for $\mathrm{C}_{22} \mathrm{H}_{20} \mathrm{FN}_{2} \mathrm{O}_{2} \mathrm{~S}_{2}\left([\mathrm{M}+\mathrm{H}]^{+}\right)$427.0945, found 427.0953.

N-Phenyl-5-(4-methylphenyl)-3-tosylthiazolidin-2-imine (5c). White solid, $\mathrm{mp} 115-117{ }^{\circ} \mathrm{C}(\mathrm{EtOAc}-\mathrm{PE})^{34}{ }^{1} \mathrm{H}$

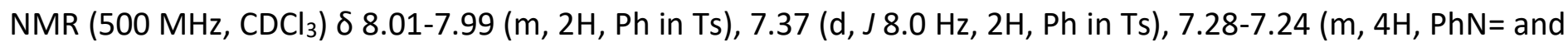
5-Ph), $7.15(\mathrm{~d}, J 8.0 \mathrm{~Hz}, 2 \mathrm{H}, 5-\mathrm{Ph}), 7.10-7.06(\mathrm{~m}, 1 \mathrm{H}, \mathrm{PhN}=), 6.81-6.79(\mathrm{~m}, 2 \mathrm{H}, \mathrm{PhN}=), 4.79(\mathrm{dd}, J 8.8,6.5 \mathrm{~Hz}, 1 \mathrm{H}$, $\mathrm{CH}_{2}$ ), 4.60 (dd, J 10.4, $6.4 \mathrm{~Hz}, 1 \mathrm{H}, \mathrm{CH}_{2}$ ), 4.03 (dd, J 10.4, $\left.8.8 \mathrm{~Hz}, 1 \mathrm{H}, \mathrm{CHS}\right), 2.50$ (s, 3H, Me in Ts), 2.35 (s, 3H, Me). ${ }^{13} \mathrm{C}$ NMR $\left(125 \mathrm{MHz}, \mathrm{CDCl}_{3}\right) \delta 152.3,150.1,144.9,138.7,134.7,133.4,129.7,129.2,129.1,128.9,127.4$, 124.3, 120.8, 56.8, 46.9, 21.7, 21.1. MS (ESI): $\left([\mathrm{M}+\mathrm{H}]^{+}\right)$423.1; HRMS (ESI) calculated for $\mathrm{C}_{23} \mathrm{H}_{23} \mathrm{~N}_{2} \mathrm{O}_{2} \mathrm{~S}_{2}\left([\mathrm{M}+\mathrm{H}]^{+}\right)$ 423.1195, found 423.1195 .

N-Phenyl-5-(2-chlorophenyl)-3-tosylthiazolidin-2-imine (5d). Viscous yellow liquid ${ }^{34} ;{ }^{1} \mathrm{H}$ NMR $(500 \mathrm{MHz}$, $\left.\mathrm{CDCl}_{3}\right) \delta 7.98(\mathrm{~d}, J 8.3 \mathrm{~Hz}, 2 \mathrm{H}, \mathrm{Ph}$ in Ts$), 7.59-7.57(\mathrm{~m}, 1 \mathrm{H}, 5-\mathrm{Ph}), 7.42-7.36(\mathrm{~m}, 3 \mathrm{H}, \mathrm{Ph}$ in Ts and PhN=), 7.30-7.27 (m, $4 \mathrm{H}, \mathrm{PhN}=$ and 5-Ph), $7.09(\mathrm{t}, J 7.4 \mathrm{~Hz}, 1 \mathrm{H}, \mathrm{PhN}=), 6.81-6.79(\mathrm{~m}, 2 \mathrm{H}, \mathrm{PhN}=), 5.21-5.19\left(\mathrm{~m}, 1 \mathrm{H}, \mathrm{CH}_{2}\right), 4.52(\mathrm{dd}$, J 10.6, $6.5 \mathrm{~Hz}, 1 \mathrm{H}, \mathrm{CH}_{2}$ ), 4.29 (dd, J 10.6, $\left.5.4 \mathrm{~Hz}, 1 \mathrm{H}, \mathrm{CHS}\right), 2.50$ (s, 3H, Me). $\left.{ }^{13} \mathrm{C} \mathrm{NMR} \mathrm{(125} \mathrm{MHz,} \mathrm{CDCl}\right) \delta 151.7$, 150.0, 145.0, 135.4, 134.6, 133.5, 129.9, 129.7, 129.24, 129.19, 129.0, 128.0, 127.6, 124.4, 120.7, 55.2, 43.5, 42.7, 21.7. $\mathrm{MS}(\mathrm{ESI}):\left([\mathrm{M}+\mathrm{H}]^{+}\right)$443.1; HRMS (ESI) calculated for $\mathrm{C}_{22} \mathrm{H}_{20} \mathrm{CIN}_{2} \mathrm{O}_{2} \mathrm{~S}_{2}\left([\mathrm{M}+\mathrm{H}]^{+}\right)$443.0649, found 443.0652.

N-Phenyl-5-(4-bromophenyl)-3-tosylthiazolidin-2-imine (5e). Colorless liquid ${ }^{34} ;{ }^{1} \mathrm{H} N M R\left(500 \mathrm{MHz} \mathrm{CDCl}_{3}\right) \delta$ 7.98 (d, J 8.3 Hz, 2H, 5-Ph), 7.49-7.46 (m, 2H, Ph in Ts), 7.37 (d, J 8.2 Hz, 2H, Ph in Ts), 7.30-7.22 (m, 4H, 5-Ph and $\mathrm{PhN}=$ ), $7.09(\mathrm{t}, J 7.4 \mathrm{~Hz}, 1 \mathrm{H}, \mathrm{PhN}=), 6.79(\mathrm{~d}, J 7.4 \mathrm{~Hz}, 2 \mathrm{H}, \mathrm{PhN}=), 4.76-4.73\left(\mathrm{~m}, 1 \mathrm{H}, \mathrm{CH}_{2}\right), 4.58(\mathrm{dd}, J 10.4,6.4$ $\mathrm{Hz}, 1 \mathrm{H}, \mathrm{CH}_{2}$ ), 4.05 (dd, J 10.4, $\left.7.9 \mathrm{~Hz}, 1 \mathrm{H}, \mathrm{CHS}\right), 2.50$ (s, 3H, Me). $\left.{ }^{13} \mathrm{C} \mathrm{NMR} \mathrm{(125} \mathrm{MHz,} \mathrm{CDCl}\right)_{3} \delta 151.6,149.9$, 145.0, 135.9, 134.6, 132.2, 129.3, 129.2, 129.0, 124.5, 122.7, 120.7, 56.6, 46.3, 21.7. MS (ESI): $\left([\mathrm{M}+\mathrm{H}]^{+}\right)$489.0; HRMS (ESI) calculated for $\mathrm{C}_{22} \mathrm{H}_{20} \mathrm{BrN}_{2} \mathrm{O}_{2} \mathrm{~S}_{2}\left([\mathrm{M}+\mathrm{H}]^{+}\right)$487.0144, found 487.0162.

$\boldsymbol{N}$-(4-Methoxyphenyl)-5-phenyl-3-tosylthiazolidin-2-imine (5f). Yellow liquid ${ }^{34} ;{ }^{1} \mathrm{H} \mathrm{NMR}\left(500 \mathrm{MHz} \mathrm{CDCl}_{3}\right) \delta$ 8.00 (d, J 8.2 Hz, 2H, Ph in Ts), 7.37-7.34 (m, 7H, Ph in Ts, PhN= and 5-Ph), 6.82-6.77 (dd, J 8.8, 8.9 Hz, 4H, PhN= 
and 5-Ph), $4.81\left(\mathrm{dd}, J\right.$ 8.3, $\left.6.6 \mathrm{~Hz}, 1 \mathrm{H}, \mathrm{CH}_{2}\right), 4.60$ (dd, J 10.3, 6.4 Hz, 1H, $\mathrm{CH}_{2}$ ), 4.06 (dd, J 10.2, 8.7 Hz, 1H, CHS), 3.78 (s, 3H, OMe), 2.49 (s, 3H, Me). $\left.{ }^{13} \mathrm{C} \mathrm{NMR} \mathrm{(125} \mathrm{MHz,} \mathrm{CDCl}{ }_{3}\right) \delta 156.6,151.7,144.8,143.4,136.7,134.9$, 129.2, 129.0, 128.7, 127.5 121.9, 114.2, 56.7, 55.4, 47.0, 21.7. MS (ESI): ([M+H] ${ }^{+}$) 439.4; HRMS (ESI) calculated for $\mathrm{C}_{23} \mathrm{H}_{23} \mathrm{~N}_{2} \mathrm{O}_{3} \mathrm{~S}_{2}\left([\mathrm{M}+\mathrm{H}]^{+}\right)$439.1145, found 439.1162 .

$\mathbf{N}$-(4-Fluorophenyl)-5-phenyl-3-tosylthiazolidin-2-imine (5g). Colorless liquid ${ }^{34} ;{ }^{1} \mathrm{H} \mathrm{NMR}\left(500 \mathrm{MHz} \mathrm{CDCl}_{3}\right) \delta$ $7.98(\mathrm{~d}, J 8.3 \mathrm{~Hz}, 2 \mathrm{H}, \mathrm{Ph}$ in Ts$), 7.38-7.34(\mathrm{~m}, 7 \mathrm{H}, \mathrm{Ph}$ in Ts and 5-Ph), 6.98-6.94 (m, 2H, PhN=), 6.778-6.75 (m, 2H, $\mathrm{PhN}=$ ), $4.82\left(\mathrm{dd}, J\right.$ 8.5, $6.5 \mathrm{~Hz}, 1 \mathrm{H}, \mathrm{CH}_{2}$ ), 4.61 (dd, J 10.4, $6.5 \mathrm{~Hz}, 1 \mathrm{H}, \mathrm{CH}_{2}$ ), 4.08 (dd, J 10.4, 8.5 Hz, 1H, CHS), 2.50 (s, 3H, Me). ${ }^{13} \mathrm{C}$ NMR $\left(125 \mathrm{MHz}_{\mathrm{CDCl}}\right.$ ) $\delta 160.7,158.8,152.4,146.1,145.0,144.5,136.4,134.7,129.3,129.2$, $129.1,128.8,127.5,122.2,122.1,115.7,115.6,56.8,47.1,21.7 . \mathrm{MS}$ (ESI): $\left([\mathrm{M}+\mathrm{H}]^{+}\right)$427.2; HRMS (ESI) calculated for $\mathrm{C}_{22} \mathrm{H}_{20} \mathrm{FN}_{2} \mathrm{O}_{2} \mathrm{~S}_{2}\left([\mathrm{M}+\mathrm{H}]^{+}\right) 427.0945$, found 427.0964 .

$\mathbf{N}$-(2-Methylphenyl)-5-phenyl-3-tosylthiazolidin-2-imine (5h). Viscous liquid; ${ }^{1} \mathrm{H} \mathrm{NMR}\left(500 \mathrm{MHz}, \mathrm{CDCl}_{3}\right) \delta 7.98$ (d, J 8.3 Hz, 2H, Ph in Ts), 7.39-7.34 (m, 7H, Ph in Ts and 5-Ph), 7.14-7.08 (m, 2H, 2-Ph), 7.01-6.98 (m, 1H, 2-Ph), $6.69(\mathrm{~d}, J 7.7 \mathrm{~Hz}, 1 \mathrm{H}, 2-\mathrm{Ph}), 4.82\left(\mathrm{dd}, J 8.2,6.5 \mathrm{~Hz}, 1 \mathrm{H}, \mathrm{CH}_{2}\right), 4.65$ (dd, J 10.4, $6.4 \mathrm{~Hz}, 1 \mathrm{H}, \mathrm{CH}_{2}$ ), 4.14 (dd, J 10.3, $8.3 \mathrm{~Hz}, 1 \mathrm{H}, \mathrm{CHS}), 2.48$ (s, 3H, Me in Ts), 1.95 (s, 3H, Me). $\left.{ }^{13} \mathrm{C} \mathrm{NMR} \mathrm{(125} \mathrm{MHz,} \mathrm{CDCl} 3\right) \delta 151.7,149.2,144.7$, 136.8, 135.2, 130.3, 129.4, 129.3, 129.0, 128.9, 128.7, 127.4, 126.3, 124.3, 119.6, 57.0, 46.8, 21.6, 17.4. MS (ESI): $\left([\mathrm{M}+\mathrm{H}]^{+}\right)$423.1; HRMS (ESI) calculated for $\mathrm{C}_{23} \mathrm{H}_{23} \mathrm{~N}_{2} \mathrm{O}_{2} \mathrm{~S}_{2}\left([\mathrm{M}+\mathrm{H}]^{+}\right)$423.1195, found 423.1184. El. Anal. calculated for $\mathrm{C}_{23} \mathrm{H}_{22} \mathrm{~N}_{2} \mathrm{O}_{2} \mathrm{~S}_{2}$ : C, 65.38; $\mathrm{H}, 5.25 ; \mathrm{N}, 6.63$; found $\mathrm{C}, 65.29 ; \mathrm{H}, 5.37 ; \mathrm{N}, 6.89$.

$\boldsymbol{N}$-(4-Bromophenyl)-5-phenyl-3-tosylthiazolidin-2-imine (5i). Viscous liquid; ${ }^{1} \mathrm{H} \mathrm{NMR}\left(500 \mathrm{MHz}, \mathrm{CDCl}_{3}\right) \delta 7.97$ (d, J 8.2 Hz, 2H, Ph in Ts), 7.43-7.34 (m, 9H, Ph in Ts, 5-Ph and 2-Ph), 6.68 (d, J 8.6 Hz, 2H, 2-Ph), 4.83 (dd, J 8.5, $6.5 \mathrm{~Hz}, 1 \mathrm{H}, \mathrm{CH}_{2}$ ), 4.62 (dd, J 10.4, $6.5 \mathrm{~Hz}, 1 \mathrm{H}, \mathrm{CH}_{2}$ ), 4.08 (dd, J 10.3, 8.6 Hz, 1H, CHS), 2.50 (s, 3H, Me). ${ }^{13} \mathrm{C} \mathrm{NMR}$ $\left(125 \mathrm{MHz}_{1} \mathrm{CDCl}_{3}\right) \delta 152.8,148.9,145.1,136.3,134.6,132.0,129.3,129.14,129.11,128.9,127.5,122.6,117.4$, 56.8, 47.1, 21.7. MS (ESI): $\left([\mathrm{M}+\mathrm{H}]^{+}\right)$487.0; HRMS (ESI) calculated for $\mathrm{C}_{22} \mathrm{H}_{20} \mathrm{BrN}_{2} \mathrm{O}_{2} \mathrm{~S}_{2}\left([\mathrm{M}+\mathrm{H}]^{+}\right)$487.0144, found 487.0124. El. Anal. calculated for $\mathrm{C}_{22} \mathrm{H}_{19} \mathrm{BrN}_{2} \mathrm{O}_{2} \mathrm{~S}_{2}$ : C, 54.21; $\mathrm{H}, 3.93 ; \mathrm{N}, 5.75$; found $\mathrm{C}, 54.25 ; \mathrm{H}, 3.68 ; \mathrm{N}, 5.68$.

$\mathbf{N}$-(4-Chlorophenyl)-5-phenyl-3-tosylthiazolidin-2-imine (5j). Viscous liquid; ${ }^{1} \mathrm{H} \mathrm{NMR}\left(500 \mathrm{MHz}, \mathrm{CDCl}_{3}\right) \delta 7.97$ (d, J $8.3 \mathrm{~Hz}, 2 \mathrm{H}, \mathrm{Ph}$ in Ts), 7.38-7.33 (m, 7H, Ph in Ts, 5-Ph and 2-Ph), 7.24-7.22 (m, 2H, 5-Ph), 6.75-6.72 (m, 2H, 2-Ph), 4.83 (dd, J 8.5, $6.5 \mathrm{~Hz}, 1 \mathrm{H}, \mathrm{CH}_{2}$ ), 4.62 (dd, J 10.3, $6.5 \mathrm{~Hz}, 1 \mathrm{H}, \mathrm{CH}_{2}$ ), 4.08 (dd, J 10.4, 8.5 Hz, 1H, CHS), 2.50 (s, 3H, Me). ${ }^{13} \mathrm{C}$ NMR $\left(125 \mathrm{MHz}, \mathrm{CDCl}_{3}\right) \delta 152.8,148.5,145.1,136.3,134.6,129.6,129.3,129.14,129.10$, 129.05, 128.9, 127.5, 122.2, 56.8, 47.1, 21.7. MS (ESI): $\left([\mathrm{M}+\mathrm{H}]^{+}\right)$443.1; HRMS (ESI) calculated for $\mathrm{C}_{22} \mathrm{H}_{20} \mathrm{ClN}_{2} \mathrm{O}_{2} \mathrm{~S}_{2}\left([\mathrm{M}+\mathrm{H}]^{+}\right)$443.0649, found 443.0635. El. Anal. calculated for $\mathrm{C}_{22} \mathrm{H}_{19} \mathrm{ClN}_{2} \mathrm{O}_{2} \mathrm{~S}_{2}: \mathrm{C}, 59.65 ; \mathrm{H}, 4.32 ; \mathrm{N}$, 6.32; found C, 59.38; H, 4.60; N, 6.34.

$\mathbf{N}$-(Isopropyl)-5-phenyl-3-tosylthiazolidin-2-imine (5k). White solid, mp 134-136 ${ }^{\circ} \mathrm{C}(\mathrm{EtOAc}-\mathrm{PE}){ }^{35} ;{ }^{1} \mathrm{H} N M R(500$ $\left.\mathrm{MHz}, \mathrm{CDCl}_{3}\right) \delta 7.93$ (d, J 8.3 Hz, 2H, Ph in Ts), 7.39-7.34 (m, 5H, Ph in Ts and 5-Ph), 7.31-7.28 (m, 2H, 5-Ph), 4.78

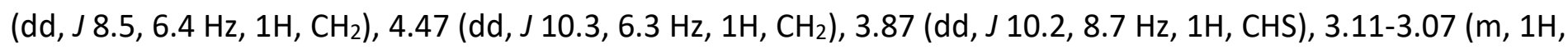
$\mathrm{CH}$ in isopropyl), 2.47 (s, 3H, Me in Ts), 1.14 (d, J $6.2 \mathrm{~Hz}, 3 \mathrm{H}, \mathrm{Me}), 1.08$ (d, J $6.2 \mathrm{~Hz}, 3 \mathrm{H}, \mathrm{Me}) .{ }^{13} \mathrm{C} \mathrm{NMR}(125 \mathrm{MHz}$, $\left.\mathrm{CDCl}_{3}\right) \delta 147.2,144.4,137.0,134.9,129.3,129.0,128.9,128.6,127.6,57.7,56.0,46.9,23.6,23.4,21.7 . \mathrm{MS}$ (ESI): $\left([\mathrm{M}+\mathrm{H}]^{+}\right) 375.1$;

$\mathbf{N}$-Cyclohexyl-5-phenyl-3-tosylthiazolidin-2-imine (5I). White solid, mp 152-154 ${ }^{\circ} \mathrm{C}\left(\right.$ EtOAc-PE) ${ }^{34} ;{ }^{1} \mathrm{H}$ NMR $(500$ $\left.\mathrm{MHz}_{1} \mathrm{CDCl}_{3}\right) \delta 7.93$ (d, J 8.3 Hz, 2H, Ph in Ts), 7.39-7.28 (m, 7H, Ph in Ts and 5-Ph), $4.78(\mathrm{dd}, J 8.5,6.3 \mathrm{~Hz}, 1 \mathrm{H}$, $\mathrm{CH}_{2}$ ), 4.47 (dd, J 10.3, 6.3 Hz, 1H, CH 2$), 3.89$ (dd, J 10.2, 8.5 Hz, 1H, CHS), 2.79-2.77 (m, 1H, CH in cyclohexyl), $2.46(\mathrm{~s}, 3 \mathrm{H}, \mathrm{Me}), 1.74-1.56(\mathrm{~m}, 6 \mathrm{H}, \mathrm{cyclohexyl}), 1.30-1.24(\mathrm{~m}, 4 \mathrm{H}, \mathrm{cyclohexyl}) .{ }^{13} \mathrm{C} \mathrm{NMR}(125 \mathrm{MHz}, \mathrm{CDCl} 3) \delta$ 147.1, 144.3, 137.1, 135.0, 129.2, 129.0, 128.9, 128.6, 127.6, 56.0, 46.9, 33.5, 33.3, 25.7, 24.4, 21.7. MS (ESI): $\left([\mathrm{M}+\mathrm{H}]^{+}\right)$415.3; HRMS (ESI) calculated for $\mathrm{C}_{22} \mathrm{H}_{27} \mathrm{~N}_{2} \mathrm{O}_{2} \mathrm{~S}_{2}\left([\mathrm{M}+\mathrm{H}]^{+}\right)$415.1508, found 415.1510.

$\boldsymbol{N}$-(n-Butyl)-5-phenyl-3-tosylthiazolidin-2-imine (5m). Viscous liquid ${ }^{34} ;{ }^{1} \mathrm{H} N M R\left(500 \mathrm{MHz}, \mathrm{CDCl}_{3}\right) \delta 7.92-7.90$ (m, 2H, Ph in Ts), 7.40-7.34 (m, 5H, Ph in Ts and 5-Ph), 7.31 (d, J 8.3 Hz, 2H, 5-Ph), 4.79 (dd, J 8.4, 6.3 Hz, 1H, 
$\mathrm{CH}_{2}$ ), 4.50 (dd, J 10.3, $6.3 \mathrm{~Hz}, 1 \mathrm{H}, \mathrm{CH}_{2}$ ), 3.93 (dd, J 10.2, 8.4 Hz, 1H, CHS), 3.24-3.19 (m, 1H, $\mathrm{CH}_{2}$ in butyl), 3.11$3.07\left(\mathrm{~m}, 1 \mathrm{H}, \mathrm{CH}_{2}\right.$ in butyl), 2.46 (s, 3H, Me in Ts), 1.53-1.49 (m, 2H, $\mathrm{CH}_{2}$ in butyl), 1.22-1.17 (m, $2 \mathrm{H}, \mathrm{CH}_{2}$ in butyl), 0.88 (t, J $7.4 \mathrm{~Hz}, 3 \mathrm{H}, \mathrm{Me}) .{ }^{13} \mathrm{C}$ NMR $\left(125 \mathrm{MHz}, \mathrm{CDCl}_{3}\right) \delta 149.3,144.4,137.0,135.1,129.1,129.0,128.6$, 127.6, 56.3, 55.9, 46.9, 32.8, 21.6, 20.4, 13.9. MS (ESI): ([M+H] $\left.]^{+}\right) 389.3 ; \mathrm{HRMS}$ (ESI) calculated for $\mathrm{C}_{20} \mathrm{H}_{25} \mathrm{~N}_{2} \mathrm{O}_{2} \mathrm{~S}_{2}$ $\left([\mathrm{M}+\mathrm{H}]^{+}\right)$389.1352, found 389.1346.

N-Benzyl-5-phenyl-3-tosylthiazolidin-2-imine (5n). White solid, mp $120-122{ }^{\circ} \mathrm{C}$ (EtOAc-PE); ${ }^{1} \mathrm{H} \mathrm{NMR}(500 \mathrm{MHz}$, $\left.\mathrm{CDCl}_{3}\right) \delta 7.86$ (d, J 8.2 Hz, 2H, Ph in Ts), 7.42-7.35 (m, 5H, Ph in benzyl), 7.29-7.24 (m, 3H, Ph in Ts and 5-Ph), 7.19 (d, J $8.1 \mathrm{~Hz}, 2 \mathrm{H}, 5-\mathrm{Ph}), 7.16-7.14(\mathrm{~m}, 2 \mathrm{H}, 5-\mathrm{Ph}), 4.86\left(\mathrm{dd}, J\right.$ 8.2, $\left.6.5 \mathrm{~Hz}, 1 \mathrm{H}, \mathrm{CH}_{2}\right), 4.56(\mathrm{dd}, J 10.3,6.3 \mathrm{~Hz}, 1 \mathrm{H}$, $\mathrm{CH}_{2}$ ), 4.44 (d, J $15.2 \mathrm{~Hz}, 1 \mathrm{H}, \mathrm{CHS}$ ), 4.36 (d, J $15.2 \mathrm{~Hz}, 1 \mathrm{H}, \mathrm{CH}_{2}-\mathrm{Ph}$ ), 4.01 (dd, J 10.2, 8.4 Hz, 1H, CH $\left.2-\mathrm{Ph}\right), 2.43$ (s, $3 \mathrm{H}, \mathrm{Me}) .{ }^{13} \mathrm{C} \mathrm{NMR}\left(125 \mathrm{MHz}, \mathrm{CDCl}_{3}\right) \delta 151.2,144.4,139.4,136.8,135.1,129.2,129.1,129.0,128.8,128.1$, 127.6, 127.5, 126.6, 59.4, 56.5, 47.2, 21.6. MS (ESI): ([M+H] $\left.]^{+}\right)$423.3; HRMS (ESI) calculated for $\mathrm{C}_{23} \mathrm{H}_{23} \mathrm{~N}_{2} \mathrm{O}_{2} \mathrm{~S}_{2}$ $\left([\mathrm{M}+\mathrm{H}]^{+}\right)$423.1195, found 423.1195. El. Anal. calculated for $\mathrm{C}_{23} \mathrm{H}_{22} \mathrm{~N}_{2} \mathrm{O}_{2} \mathrm{~S} 2: \mathrm{C}, 65.38 ; \mathrm{H}, 5.25 ; \mathrm{N}, 6.63$ found C, $65.41 ; H, 5.32 ; \mathrm{N}, 6.49$.

$\mathbf{N}$-( $\alpha$-Methylbenzyl)-5-phenyl-3-tosylthiazolidin-2-imine (5o). Viscous liquid; ${ }^{1} \mathrm{H} \mathrm{NMR}\left(500 \mathrm{MHz}, \mathrm{CDCl}_{3}\right) \delta 7.96$ (d, J $8.3 \mathrm{~Hz}, 1 / 4 \times 2 \mathrm{H}), 7.90(\mathrm{~d}, J 8.2 \mathrm{~Hz}, 3 / 4 \times 2 \mathrm{H}), 7.40-7.32(\mathrm{~m}, 5 \mathrm{H}), 7.30-7.20(\mathrm{~m}, 5+1 / 4 \times 2 \mathrm{H}), 7.18-7.17(\mathrm{~m}$, $3 / 4 \times 2 \mathrm{H}), 4.85-4.75(\mathrm{~m}, 1 \mathrm{H}), 4.55-4.49(\mathrm{~m}, 1 \mathrm{H}), 4.14-4.10(\mathrm{~m}, 1 \mathrm{H}), 3.98-3.92(\mathrm{~m}, 1 \mathrm{H}), 2.46(\mathrm{~s}, 1 / 4 \times 3 \mathrm{H}), 2.44(\mathrm{~s}$, $3 / 4 \times 3 \mathrm{H}), 1.43(\mathrm{~d}, J 6.5 \mathrm{~Hz}, 3 / 4 \times 3 \mathrm{H}), 1.38(\mathrm{~d}, J 6.5 \mathrm{~Hz}, 1 / 4 \times 3 \mathrm{H}) .{ }^{13} \mathrm{C} \mathrm{NMR}\left(125 \mathrm{MHz}, \mathrm{CDCl}_{3}\right) \delta 148.9,145.1,145.0$, $144.4,144.3,136.9,136.7,135.3,129.2,129.05,129.00,128.9,128.7,128.6,128.2,128.1,127.6,127.5,126.6$, $126.57,126.5,126.4,65.5,65.4,56.2,56.0,47.3,47.1,25.1,24.6,21.6 . \mathrm{MS}$ (ESI): ([M+H] ${ }^{+}$) 437.1; HRMS (ESI) calculated for $\mathrm{C}_{24} \mathrm{H}_{25} \mathrm{~N}_{2} \mathrm{O}_{2} \mathrm{~S}_{2}\left([\mathrm{M}+\mathrm{H}]^{+}\right)$437.1352, found 437.1350. El. Anal. calculated for $\mathrm{C}_{24} \mathrm{H}_{24} \mathrm{~N}_{2} \mathrm{O}_{2} \mathrm{~S}_{2}: \mathrm{C}_{,} 66.03$; $H, 5.54 ; N, 6.42$ found C, 65.88; H, 5.31; N, 6.25 .

$\boldsymbol{N}$-( $\boldsymbol{\alpha}$-Naphthyl)-5-phenyl-3-tosylthiazolidin-2-imine (5p). Yellow solid, mp $133-135{ }^{\circ} \mathrm{C}(\mathrm{EtOAc}-\mathrm{PE})^{33} ; \delta 8.03(\mathrm{~d}$, J $8.3 \mathrm{~Hz}, 2 \mathrm{H}$, naphthyl), 7.80 (d, J $8.2 \mathrm{~Hz}, 1 \mathrm{H}, \mathrm{Ph}$ in Ts), 7.58 (d, J $8.3 \mathrm{~Hz}, 1 \mathrm{H}, \mathrm{Ph}$ in Ts), 7.49-7.45 (m, 2H), 7.39$7.30(\mathrm{~m}, 9 \mathrm{H}, 5-\mathrm{Ph}, \mathrm{Ph}$ in Ts and naphthyl), 6.87 (d, J $7.1 \mathrm{~Hz}, 1 \mathrm{H}, 5-\mathrm{Ph}), 4.84\left(\mathrm{dd}, J\right.$ 8.3, $\left.6.5 \mathrm{~Hz}, 1 \mathrm{H}, \mathrm{CH}_{2}\right), 4.72(\mathrm{dd}$, J 10.4, 6.4 Hz, 1H, CH 2 ), 4.21 (dd, J 10.3, 8.4 Hz, 1H, CHS), 2.52 (s, 3H, Me). ${ }^{13} \mathrm{C} \mathrm{NMR} \mathrm{(125} \mathrm{MHz,} \mathrm{CDCl} 3$ ) $\delta 152.4$, 146.9, 144.8, 136.6, 135.4, 134.1, 129.5, 129.13, 129.05, 128.8, 127.7, 127.5, 126.2, 125.7, 125.3, 124.5, 123.9, 114.7, 56.9, 46.9, 21.7. MS (ESI): $\left([\mathrm{M}+\mathrm{H}]^{+}\right)$459.1; HRMS (ESI) calculated for $\mathrm{C}_{26} \mathrm{H}_{23} \mathrm{~N}_{2} \mathrm{O}_{2} \mathrm{~S}_{2}\left([\mathrm{M}+\mathrm{H}]^{+}\right) 459.1195$, found 459.1203.

N,5-Diphenyl-3-(phenylsulfonyl)thiazolidin-2-imine (5q). Viscous liquid ${ }^{34} ;{ }^{1} \mathrm{H} N M R\left(500 \mathrm{MHz}, \mathrm{CDCl}_{3}\right) \delta 8.12(\mathrm{~d}$, J $7.5 \mathrm{~Hz}, 2 \mathrm{H}, \mathrm{Ph}$ in Ts), 7.71 (t, J $7.5 \mathrm{~Hz}, 1 \mathrm{H}, \mathrm{Ph}$ in Ts), 7.59 (t, J 7.8 Hz, 2H, Ph in Ts), 7.37-7.34 (m, 5H, 5-Ph), 7.29-7.25 (m, $2 \mathrm{H}, \mathrm{PhN}=), 7.08(\mathrm{t}, J 7.4 \mathrm{~Hz}, 1 \mathrm{H}, \mathrm{PhN}=), 6.78(\mathrm{~d}, J 7.6 \mathrm{~Hz}, 2 \mathrm{H}, \mathrm{PhN}=), 4.83(\mathrm{dd}, J 8.4,6.5 \mathrm{~Hz}, 1 \mathrm{H}$, $\mathrm{CH}_{2}$ ), 4.64 (dd, J 10.4, $\left.6.4 \mathrm{~Hz}, 1 \mathrm{H}, \mathrm{CH}_{2}\right), 4.11$ (dd, J 10.4, $\left.8.5 \mathrm{~Hz}, 1 \mathrm{H}, \mathrm{CHS}\right) .{ }^{13} \mathrm{C} \mathrm{NMR}\left(125 \mathrm{MHz}, \mathrm{CDCl}_{3}\right) \delta 152.1$, $149.9,137.7,136.5,133.9,129.14,129.07,128.98,128.8,128.6,127.5,124.4,120.7,56.8,47.1$. MS (ESI): $\left([\mathrm{M}+\mathrm{H}]^{+}\right) 395.1$.

\section{Acknowledgements}

This work was supported by the Natural Science Foundation of Zhejiang Provincial (LY15B020004), the Natural Science Foundation of China (21602197) and the Open Research Fund Program of Collaborative Innovation Center of Membrane Separation and Water Treatment (2016YB07).

\section{Supplementary Material}

Copies of ${ }^{1} \mathrm{H}$ and ${ }^{13} \mathrm{C}$ NMR spectra for all isolated compounds. 


\section{References}

1. Narayanam, J. M. R.; Stephenson, C. R. J. Chem. Soc. Rev. 2011, 40, 102. https://doi.org/10.1039/B913880N

2. Lu, Z.; Yoon, T. P. Angew. Chem. Int. Ed. 2012, 51, 10329. https://doi.org/10.1002/anie.201204835

3. Shi, L.; Xia, W. Chem. Soc. Rev. 2012, 41, 7687. https://doi.org/10.1039/c2cs35203f

4. Xuan, J.; Xia, X.; Zeng, T.; Lu, L.; Xiao, W. Angew. Chem. Int. Ed. 2014, 53, 5653. https://doi.org/10.1002/anie.201400602

5. Shaw, M. H.; Twilton, J.; MacMillan, D. W. C. J. Org. Chem. 2016, 81, 6898. https://doi.org/10.1021/acs.joc.6b01449

6. Ciamician, G. Science 1912, 36, 385. https://doi.org/10.1126/science.36.926.385

7. Nicewicz, D. A.; MacMillan, D. W. C. Science 2008, 322, 77. https://doi.org/10.1126/science.1161976

8. Janvier, P.; Sun, X. W.; Bienayme, H.; Zhu, J. P. J. Am. Chem. Soc. 2002, 124, 2560. https://doi.org/10.1021/ja017563a

9. Krasavin, K. Eur. J. Med. Chem. 2015, 97, 525. https://doi.org/10.1016/i.ejmech.2014.11.028

10. Evans, D. A.; Downey, C. W.; Hubbs, J. L. J. Am. Chem. Soc. 2003, 125, 8706. https://doi.org/10.1021/ja035509j

11. Murugana, R.; Anbazhaganb, S.; Narayanan, S. S. Eur. J. Med. Chem. 2009, 44, 3272. https://doi.org/10.1016/j.ejmech.2009.03.035

12. Kondo, M.; Nishi, T.; Hatanaka, T.; Funahashi, Y.; Nakamura, S. Angew. Chem. Int. Ed. 2015, 54, 8198. https://doi.org/10.1002/anie.201503098

13. Wang, C.; Qin, J.; Shen, X.; Riedel, R.; Harms, K.; Meggers, E. Angew. Chem. Int. Ed. 2016, 55, 685. https://doi.org/10.1002/anie.201509524

14. Busacca, C. A.; Bartholomeyzik, T.; Cheekoori, S.; Grinberg, N.; Lee, H.; Ma, S.; Saha, A.; Shen, S.; Senanayake, C. H. J. Org. Chem. 2008, 73, 9756.

https://doi.org/10.1021/jo8024384

15. Booker-Milburn, K. I.; Guly, D. J.; Cox, B.; Procopiou, P. A. Org. Lett. 2003, 5, 3313. https://doi.org/10.1021/ol035374m

16. Zhou, L.; Zhou, J.; Tan, C. K.; Chen, J.; Yeung, Y. Y. Org. Lett. 2011, 13, 2448. https://doi.org/10.1021/ol2006902

17. Chen, J.; Yu, W.; Wei, Y.; Li, T.; Xu, P. J. Org. Chem. 2017, 82, 243. https://doi.org/10.1021/acs.joc.6b02377

18. Prasad, B. A. B.; Pandey, G.; Singh,V. K. Tetrahedron Lett. 2004, 45, 1137. https://doi.org/10.1016/i.tetlet.2003.12.015

19. Yadav, V. K.; Sriramurthy, V. J. Am. Chem. Soc. 2005, 127, 16366. https://doi.org/10.1021/ja055664t

20. Ghorai, M. K.; Das, K.; Kumar, A.; Ghosh, K. Tetrahedron Lett. 2005, 46, 4103. https://doi.org/10.1016/j.tetlet.2005.04.006

21. Ghorai, M. K.; Ghosh, K.; Das, K. Tetrahedron Lett. 2006, 47, 5399. 
https://doi.org/10.1016/i.tetlet.2005.04.006

22. Wu, J.; Sun, X.; Xia, H. G. Tetrahedron Lett. 2006, 47, 1509. https://doi.org/10.1016/j.tetlet.2006.01.021

23. Li, R.; Jiang, H.; Liu, W. Y.; Gu, P. M.; Li, X. Q. Chin. Chem. Lett. 2014, 25, 583. https://doi.org/10.1016/j.cclet.2014.01.020

24. Hsueh, N.; Clarkson, G. J.; Shipman, M. Org. Lett. 2015, 17, 3632. https://doi.org/10.1021/acs.orglett.5b01777

25. Huang, C.Y.; Doyle, A. G. Chem. Rev. 2014, 114, 8153. https://doi.org/10.1021/cr500036t

26. Kuznetsov, M. A.; Kuznetsova, L. M.; Pankova, A. S. Tetrahedron Lett. 2016, 57, 3575. https://doi.org/10.1016/j.tetlet.2016.06.116

27. Sun, H.; Yang, C.; Lin, R.; Xia, W. Adv. Synth. Catal. 2014, 356, 2775. https://doi.org/10.1002/adsc.201400476

28. Chamchaang, W.; Pinhas, A. R. J. Org. Chem. 1990, 55, 2943. https://doi.org/10.1021/jo00296a070

29. Li, X.; Yang, X.; Chang, H.; Li, Y.; Ni, B.; Wei, W. Eur. J. Org. Chem. 2011, 3122. https://doi.org/10.1002/ejoc.201100270

30. Gandhi, S.; Bisai, A.; Prasad, B. A. B.; Singh, V. K. J. Org. Chem. 2007, 72, 2133. https://doi.org/10.1021/jo062564c

31. Abe, T.; Takeda, H.; Miwa, Y.; Yamada, K.; Yanada, R.; Ishikura, M. Helv. Chim. Acta 2010, 93, 233. https://doi.org/10.1002/hlca.200900214

32. Nadir, U. K.; Joshi, S. Indian J. Chem. B 2003, 42B, 1760.

33. Gao, L.; Fu, K.; Zheng, G. RSC Adv. 2016, 6, 47192. https://doi.org/10.1039/C6RA04923K

34. Bhattacharyya, A.; Kavitha, C. V.; Ghorai, M. K. J. Org. Chem. 2016, 81, 6433. https://doi.org/10.1021/acs.joc.6b01551

35. Khalaj, A.; Khalaj, M. J. Chem. Res. 2016, 40, 445. https://doi.org/10.3184/174751916X14656621014049 